\title{
Autonomous Aerobraking Development Software: Phase One Performance Analysis at Mars, Venus, and Titan
}

\author{
Robert W. Maddock ${ }^{1}$ and Angela Bowes ${ }^{2}$ \\ NASA Langley Research Center, Hampton, VA, 23681 \\ Richard W. Powell ${ }^{3}$ \\ Analytical Mechanics Associates, Inc., Hampton, VA, 23666 \\ Jill L. H. Prince ${ }^{4}$ \\ NASA Langley Research Center, Hampton, VA, 23681 \\ and \\ Alicia Dwyer Cianciolo ${ }^{5}$ \\ NASA Langley Research Center, Hampton, VA, 23681
}

\begin{abstract}
When entering orbit about a planet or moon with an appreciable atmosphere, instead of using only the propulsion system to insert the spacecraft into its desired orbit, aerodynamic drag can be used after the initial orbit insertion to further decelerate the spacecraft. Several past NASA missions have used this aerobraking technique to reduce the fuel required to deliver a spacecraft into a desired orbit. Aerobraking was first demonstrated at Venus with Magellan in 1993 and then was used to achieve the science orbit of three Mars orbiters: Mars Global Surveyor in 1997, Mars Odyssey in 2001, and Mars Reconnaissance Orbiter in 2006. Although aerobraking itself reduces the propellant required to reach a final low period orbit, it does so at the expense of additional mission time to accommodate the aerobraking operations phase (typically 3-6 months), a large mission operations staff, and significant Deep Space Network (DSN) coverage. By automating ground based tasks and analyses associated with aerobraking and moving these onboard the spacecraft, a flight project could save millions of dollars in operations staffing and DSN costs (Ref. 1).
\end{abstract}

\section{Nomenclature}

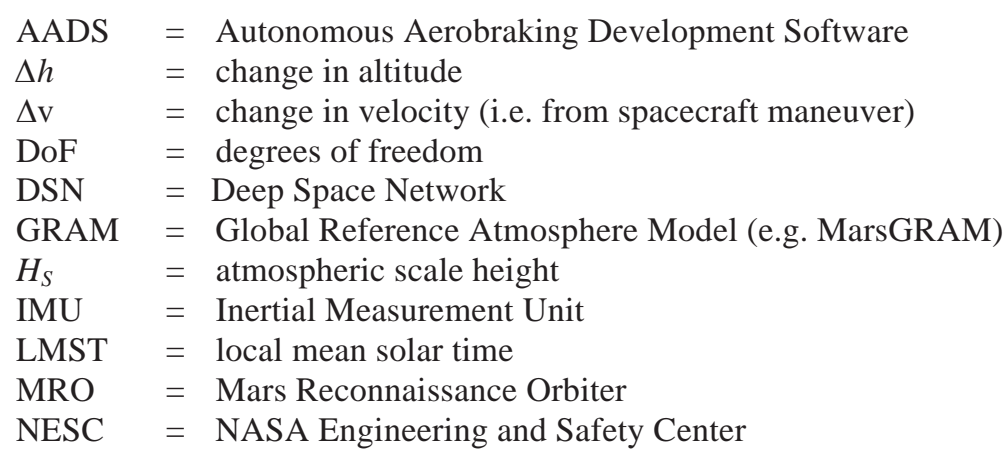

\footnotetext{
${ }^{1}$ Senior Engineer, Atmospheric Flight and Entry Systems Branch, 1 N. Dryden St./MS 489, Senior Member AIAA.

${ }^{2}$ Senior Engineer, Atmospheric Flight and Entry Systems Branch, 1 N. Dryden St./MS 489, Associate Member AIAA.

${ }^{3}$ Insert Job Title, Atmospheric Flight and Entry Systems Branch, 1 N. Dryden St./MS 489, Associate Fellow AIAA.

${ }_{5}^{4}$ Senior Engineer, Atmospheric Flight and Entry Systems Branch, 1 N. Dryden St./MS 489, Senior Member AIAA.

${ }^{5}$ Senior Engineer, Atmospheric Flight and Entry Systems Branch, 1 N. Dryden St./MS 489, Senior Member AIAA.
} 


$\begin{array}{ll}\text { ORT } & =\text { Operational Readiness Test } \\ \text { POST2 } & =\text { Program to Optimize Simulated Trajectories II } \\ \dot{q} & =\text { freestream heat rate } \\ \rho & =\text { atmospheric density }\end{array}$

\section{Introduction}

The concept of autonomous aerobraking has been studied in depth over the last decade (Ref. 2-6). The NASA Engineering and Safety Center (NESC) is currently sponsoring an Autonomous Aerobraking Capability Assessment with the main goal being to determine the feasibility of implementing autonomous aerobraking onboard a spacecraft. This assessment has culminated in the Autonomous Aerobraking Development Software (AADS). The ultimate responsibility of the AADS system is to calculate maneuvers required to maintain a spacecraft's trajectory within a desired operational corridor during successive atmospheric passes, ensuring both desired aerobraking performance as well as spacecraft safety.

The AADS is a suite of models and algorithms intended to test the feasibility of an autonomous aerobraking system. For this NESC study, the AADS system is being developed and tested for application at Mars, Venus, and Saturn's moon Titan. The AADS for application at Mars and Titan consists of three distinct modules: (1) the Ephemeris Estimator which processes spacecraft Inertial Measurement Unit (IMU) acceleration data to estimate current spacecraft states and then also propagate forward in time to predict future spacecraft states, (2) the Atmosphere Estimator which processes spacecraft acceleration data along with Ephemeris Estimator state data to estimate the atmosphere's density and scale height, and (3) the Maneuver Estimator which processes data from both the Ephemeris and Atmosphere Estimators to determine the maneuver, if required, to keep the spacecraft within the desired operational corridor. The AADS for Venus also includes a fourth module containing models to predict the maximum temperature the spacecraft will encounter during the atmospheric pass.

An overview of the AADS system is provided here, as well as a summary of the Phase 1 performance analyses for a representative Mars aerobraking mission using a freestream heat rate corridor, a Venus aerobraking mission using a solar panel temperature corridor, and a Titan aerobraking mission using an altitude corridor. Simulation analyses were run using the Program to Optimize Simulated Trajectories II (POST2) (Ref. 7-8) software integrated with the AADS system. Planned Phase 2 activities will also be discussed, and a status on the Phase 2 development and analyses will also be provided.

This paper expands upon information and data provided in a previous publication (Ref. 9). Much of what is provided here, along with additional information, is also included in the NESC AADS Phase 1 Final Report (Ref. $10)$.

\section{Phase 1 AADS}

The ultimate responsibility of the AADS system is to calculate maneuvers required to maintain a spacecraft's trajectory within a desired operational corridor during successive atmospheric passes, ensuring both desired aerobraking performance as well as spacecraft safety. Therefore, as complex as the internal AADS modules may be, the main purpose of the AADS can be summarized to simply output a maneuver vector and execution time to the spacecraft. With these pieces of information, the spacecraft can autonomously execute maneuvers at apoapsis to correct its periapsis altitude such that its design parameters are maintained within the specified corridor (e.g. heat rate, temperature, dynamic pressure or altitude). In addition, the AADS outputs the atmospheric entry/exit state estimates so that the spacecraft can properly slew to aerobraking configuration and begin and end its atmospheric data collection at the appropriate times.

The required AADS input data is passed into AADS through two data structures; the first includes parameters which are likely to change between AADS calls (e.g. spacecraft acceleration data), and the second which contains data not likely to change between AADS calls, but which may be changed and uploaded to the spacecraft during the weekly ground update cycle (e.g. corridor boundaries and target). Onboard a spacecraft, the AADS software will not always be running, but instead is called once per orbit, typically at some time after an atmospheric pass ends and prior to the next apoapsis. At each AADS call, all calculations are performed and the required data are then passed back to the spacecraft through a separate data structure. The AADS software process can then be placed in stand-by mode or terminated until the next AADS function call in order to free up spacecraft resources for other activities. Some AADS data does need to be preserved between AADS calls (e.g. Ephemeris Estimator current state prediction 
and Atmosphere Estimator atmosphere archive data), so a small amount of the spacecraft memory will need to be allocated and preserved while AADS is not running.

It is important to note that if a maneuver is commanded, but for some reason is not executed (e.g. due to some issue with the spacecraft or some other operational consideration), this does not affect the AADS in any way. Each call to AADS is distinct and independent with only limited information being passed from one call to the next. All maneuver (and drag pass) information is passed through the spacecraft IMU acceleration data so any off-nominal operations, either deliberate or due to a spacecraft or commanding error (e.g. the complete elimination of a maneuver), will be manifested in the acceleration data the AADS is provided during the next call. In otherwords, the AADS will only act upon the information (i.e. IMU acceleration data) it receives.

The AADS has been developed for feasibility testing using generalized spacecraft models based on the Mars Reconnaissance Orbiter (MRO). When a flight vehicle is selected for AADS implementation, the simulation aerodynamic and AADS thermal models must be adapted to that specific vehicle and revalidated. The atmosphere estimation and ephemeris estimation models are not vehicle specific, but only body specific, while the maneuver calculations are independent of both the vehicle and central body.

\section{Phase 1 Autonomous Aerobraking Simulation and Results}

\section{A. Mission Runout Baseline}

A reference mission design at each destination is required as a basis of comparison to assess the performance of autonomous aerobraking. The mission runout is a ground based simulation of a reference mission designed to achieve the final desired orbit conditions via aerobraking while maintaining the mission operational constraints and the margin required for the spacecraft design limits (Ref. 11). Desired final orbit conditions can include altitude, inclination, argument of periapsis, and longitude of the ascending node required to attain a specific local mean solar time (LMST) orientation, or combinations of the above. Spacecraft design and mission operational constraints may consist of spacecraft thermal and structural limits, such as freestream heat rate, solar array temperature, dynamic pressure, power, attitude, and capability to handle atmospheric density fluctuations, as well as orbit lifetime requirements, maneuver frequency restrictions, maneuver magnitude limitations, and required propellant remaining post aerobraking to achieve mission objectives.

The mission runout begins after walk-in (a series of orbits intended to incrementally lower the spacecraft periapsis into the sensible atmosphere) and lasts until the final desired orbit conditions are achieved. A corridor is determined based on a heat rate indicator, temperature, dynamic pressure or altitude to keep the spacecraft within the appropriate design margins. Maneuvers are performed at apoapsis that raise or lower periapsis to maintain the spacecraft within the pre-determined corridor. The upper limit of the corridor is determined by the required operational constraint margin to ensure spacecraft safety, and therefore defines the maximum aerobraking rate (i.e. shortest duration) that can be achieved within that constraint margin. Generally, for any of these corridor constraints, with the exception of an altitude constraint, the lower the spacecraft is in the corridor, the higher the altitude (and therefore typically lower the density) of the atmospheric passes and the lower the delta-v from aerodynamic drag, resulting in an overall increase in aerobraking mission duration. The lower corridor limit may be set to reduce the frequency of maneuvers required to stay within the corridor and/or to maintain the maneuver magnitudes above some minimum threshold. A particular lower corridor limit may also be required to ensure the aerobraking rate is such that the desired final orbit conditions can be reached by a certain time. For instance, in the case where there is a desired final orbit LMST, the initial orbit node must have enough time to precess with respect to the Sun in order to achieve the desired LMST. The amount of time required for the precession varies as a function of the initial orbit conditions, current orbit conditions, central body, gravity, atmospheric environment, and other forces such as third body perturbations and solar radiation pressure. Aerobraking either too quickly or too slowly could cause the final orbit apoapsis altitude to be reached at a different LMST than required. A corridor based on periapsis altitude can also be used, in which case the roles of the upper and lower corridor constraints are reversed (i.e., lower corridor limit defines the maximum aerobraking rate rather than the upper corridor limit since the lower the spacecraft is in the corridor means the atmospheric passes are lower and deeper within the atmosphere).

The corridor limits can change as a function of time since the specific conditions that the spacecraft is experiencing are a function of orbit geometry. A maneuver target, specified as a percentage of the corridor width, is set and can vary with time or orbit geometry as well. The minimum amount of time allowed between maneuvers is also set (once per week for Mars, once per day for Venus, and once per orbit for Titan). Whether or not a maneuver is performed when it is "allowed" is based on predicting ahead by the minimum time between maneuvers plus one 
additional day at Mars and Venus, or in the case of Titan, by predicting ahead two orbits. If a corridor violation occurs at any time during the predicted time period, a maneuver will be performed at the next allowable apoapsis.

Operationally, the mission runout is used to establish the actual spacecraft flight design corridor each week and can be adjusted if necessary during the flight to accommodate observed atmosphere fluctuations. The daily operations are used to determine any required periapsis adjust maneuvers to maintain the spacecraft within the design corridor.

\section{B. A Cycle in the life of AADS}

This section will focus on stepping through the AADS operation, at a high level, to better understand how the various models work together to successfully execute an autonomous aerobraking mission while also insuring spacecraft safety. The AADS is called just once during each orbit, at some time after atmospheric exit and prior to the next apoapsis, allowing the spacecraft sufficient time to complete any onboard tasks, including execution of the AADS software and preparations for any maneuver commanded by AADS.

The first processes executed during AADS operation involve the Ephemeris Estimator. This module's first responsibility is for estimating, through integration, the current spacecraft state. This integration begins at either the state provided for initialization (from a ground update), or from the last state propagated to by the Ephemeris Estimator and stored in memory up, and continues to the current epoch. Included in this state integration are all applicable models (central body gravity field, 3rd body gravitational effects and solar radiation pressure). The Ephemeris Estimator also integrates through all IMU acceleration data provided during both maneuvers and atmospheric passes. This is the state the Ephemeris Estimator then stores away to use as its initial state during the next AADS call, if a new initialization state is not provided. However, the Ephemeris Estimator does not stop here. The spacecraft state continues to be propagated forward through the next apoapsis, assuming no maneuver, and up to and through the next atmospheric pass, although the atmosphere itself is not modeled. This is done in order to first provide predicted apoapsis conditions for execution of a maneuver, if needed, and secondly, to provide state and altitude information for the current/previous atmosphere pass to the Atmosphere Estimator.

At this point, the AADS calls the Atmosphere Estimator (Ref. 12). The Atmosphere Estimator utilizes IMU acceleration data provided by the spacecraft, along with state and altitude information provided by the Ephemeris Estimator, to estimate the atmospheric conditions (density and scale height) during the previous pass. This information is then added to an archive where atmospheric estimates are stored for each pass. This archive data is then used to predict both the density and scale height of the next periapsis, along correlations of these predictions.

Once the Ephemeris and Atmosphere Estimators have calculated their estimates for the conditions at the spacecraft's next periapsis, the Maneuver Estimator can now determine whether or not a maneuver is required, and if so, what that maneuver should be. Figure 1 illustrates this process through the following steps:

Step 1: Calculate the predicted operational corridor location (e.g. heat rate, temperature, altitude, etc.) based on the information provided by the Ephemeris and Atmosphere Estimators.

Step 2: If the predicted location is within the corridor, do nothing. Note that this does not guarantee that the true corridor location will be within the corridor. If the AADS has not yet diverged significantly, the difference between the predicted and true corridor location should be small, certainly close enough not to trigger any immediate action.

Step 3: If the predicted location is outside of the corridor, a change in altitude is determined to place the next spacecraft periapsis at the desired corridor target.

Step 4: This change in altitude is added to the spacecraft's current orbit semi-major axis and a new velocity at apoapsis is determined. The difference between this new apoapsis velocity and the estimate of the next apoapsis velocity provided by the Ephemeris Estimator is the required maneuver magnitude. This value is positive for a periapsis raise (e.g. decrease freestream heat rate) and negative for a periapsis lowering (e.g. increase freestream heat rate). The maneuver direction is estimated to be that of the pre-maneuver velocity vector at apoapsis. Since these maneuvers are typically small $(<0.5 \mathrm{~m} / \mathrm{s})$, this assumption works well, even when considering a finite burn.

At this time, the AADS software process can be placed in stand-by mode or terminated until the next AADS function call in order to free up spacecraft resources for other activities. As previously mentioned, onboard a spacecraft, the AADS software will not always running, but instead is called once per orbit, typically at some time after an atmospheric pass ends and prior to the next apoapsis. If a maneuver is executed, the associated IMU acceleration data is collected and stored, along with the subsequent atmospheric pass IMU acceleration data, and provided to the AADS at the next call. This process is then repeated each orbit. Ideally, this would be done

4

American Institute of Aeronautics and Astronautics 
completely autonomously, with interactions from the ground no more frequent that once per week. At those times, any changes in the AADS parameters (e.g. corridor and/or target) could be made, along with an Ephemeris Estimator initialization state update.

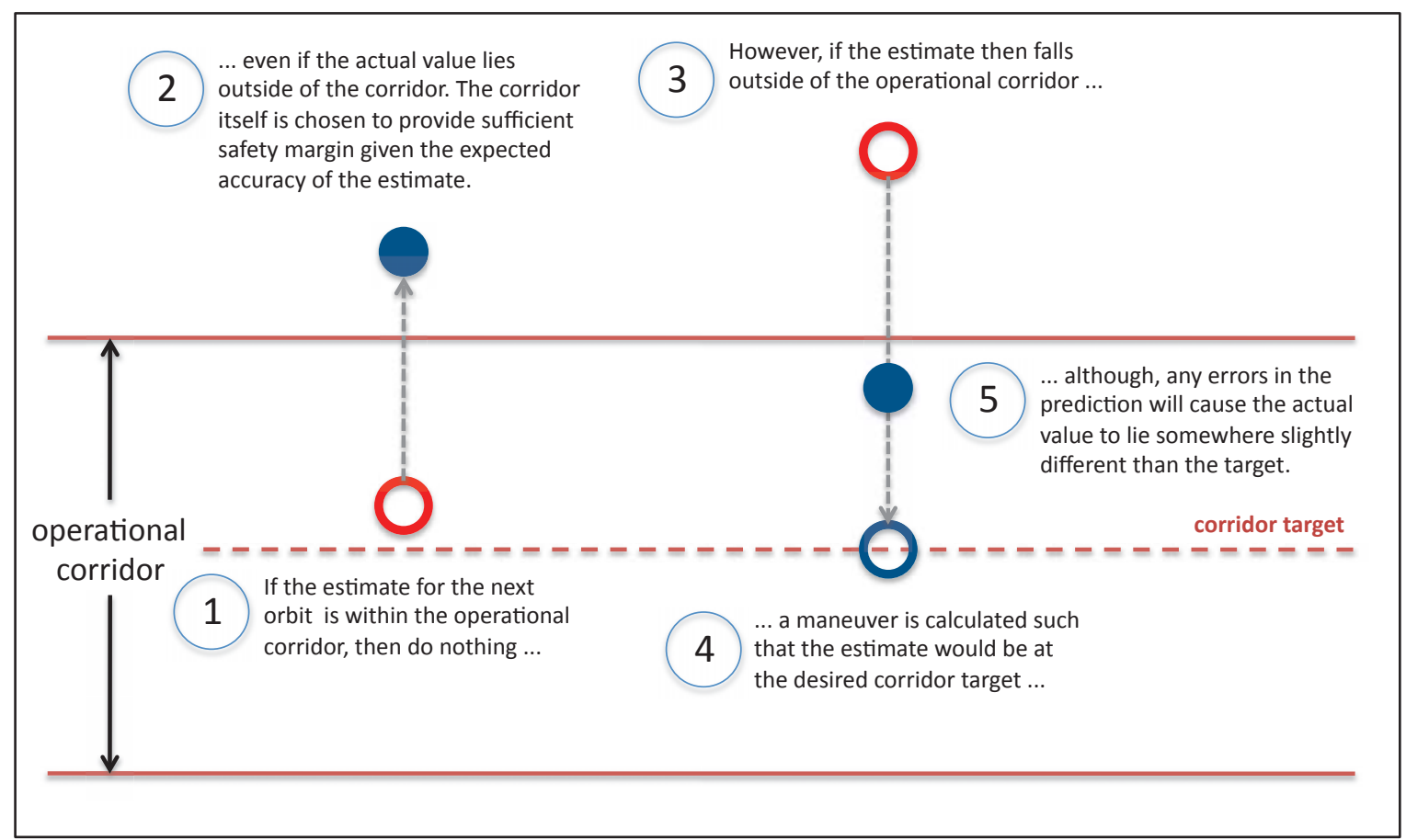

Figure 1. AADS execution process with respect to the operational corridor

\section{AADS Performance: POST2 Analyses}

The key requirement to meet for successful AADS operation is spacecraft safety throughout the main aerobraking mission. To demonstrate that AADS can accomplish this task, a set of aerobraking simulation analyses were successfully completed for each destination: Mars, Venus and Titan. The AADS POST2 simulation environment is very similar to that developed for the mission runout analyses and takes advantage of the same user defined environments and models to define the "real world", as well as the same aerobraking termination conditions. In the AADS POST2 analyses, AADS is used to predict the upcoming atmospheric pass conditions, estimate the spacecraft's location with respect to the operational corridor, and if necessary, command the spacecraft to execute a maneuver at the following apoapsis in order to achieve a specified target within the corridor during the next pass. Unlike the mission runout analyses, the AADS operation allows these maneuvers to occur on each and every orbit, if necessary. These operations are all done autonomously within the AADS software and the maneuver commands are passed back to POST2 where their execution is simulated as if AADS were running onboard the spacecraft. Updates to AADS, particularly a new "truth" state for initialization of the Ephemeris Estimator, are also simulated. These updates would be used in mission operations to periodically re-initialize the Ephemeris Estimator with a new truth state, update corridor and corridor target parameters, etc. The goal of these analyses is to show that the AADS system can provide sufficient performance (with margin) without interaction from the ground for at least one week.

Because of the high level of flexibility and modularity of POST2, as well as its extensive heritage and flight validation, it was possible to integrate the AADS code with POST2 in a way that is very "flight-like". In this simulation environment, POST2 takes on the role of modeling the physical environment as well as standing in as the spacecraft itself, where AADS is then executed through the POST2 flight software interface, in much the same way as it would be implemented onboard a spacecraft. The needed interface data structures are created on the spacecraft/POST2 side and passed into AADS. As would be the case onboard the spacecraft, the AADS code has no other interface to POST2 or the "outside world", and vice versa, except through this data structure interface. Once integrated, the POST2 and AADS code are compiled into a single executable which is then run using the POST2 user interface.

With the AADS software successfully integrated into the POST2 simulation environment, AADS performance has been assessed for application at Mars, Venus, and Titan (using 3-DoF). The AADS POST2 simulation utilizes 
the same planetary, atmosphere, gravity, spacecraft and aerodynamics models as the mission runout. For the initial state for the AADS simulation; however, the 7 th orbit of the mission runout is extracted from the mission runout results and used as the simulation initial state in order to allow data from the first seven atmospheric passes to be used for building the atmosphere archive needed by the Atmosphere Estimator. (During actual operations, this archive would likely be constructed during the "walk-in" phase, while there is still ground interaction and prior to initiation of the AADS system.) The same operational corridors as the mission runout analyses are also used for the AADS simulation; however, because this system is fully autonomous, and maneuvers are allowed to occur at any apoapsis, the corridor target selection may differ from that of the mission runout analyses.

\section{AADS Module Performance (at Mars)}

The Ephemeris Estimator performance can best be assessed by examining how well the AADS module estimates the previous periapsis state as compared to POST2 "truth." Figure 2 illustrates this performance at Mars, in terms of the differences in estimated periapsis time, when assuming a perturbed atmosphere and ground updates once per week, while Fig. 3 illustrates this performance in terms of estimated periapsis altitude. At the start of the simulation, and immediately following each ground update, when the Ephemeris Estimator is initialized with a POST2 "truth" state, the performance is quite good, showing excellent agreement (sub-second and meter level) between the Ephemeris Estimator and POST2 estimates. As time progresses, the Ephemeris Estimator propagation begins to drift (due to differences in integrators, etc.), as well as a build up of error from the lack of precision (i.e. data rate) in the IMU acceleration data provided by the spacecraft (during both the atmospheric pass and maneuver). Eventually, these effects cause the Ephemeris Estimator estimates to begin to diverge (less than 10 seconds and sub-kilometer).

The Atmosphere Estimator performance can be assessed by comparing the density and scale height estimates for the next periapsis against the "true" MarsGRAM-2010 atmosphere model values. Since the Atmosphere Estimator is attempting to fit a mean curve to the acceleration / density prediction, it is appropriate to make this comparison using an AADS run with a nominal atmosphere. Figure 4 shows, the Atmosphere Estimator density prediction is generally within $10 \%$, and the scale height within $\sim 1 \mathrm{~km}(\sim 15 \%)$, of the actual provided by the MarsGRAM-2010 model. The difference in the scale height estimates seen in Fig. 5 can be tied to the drift in the Ephemeris Estimator periapsis time and altitude (profile) estimates.

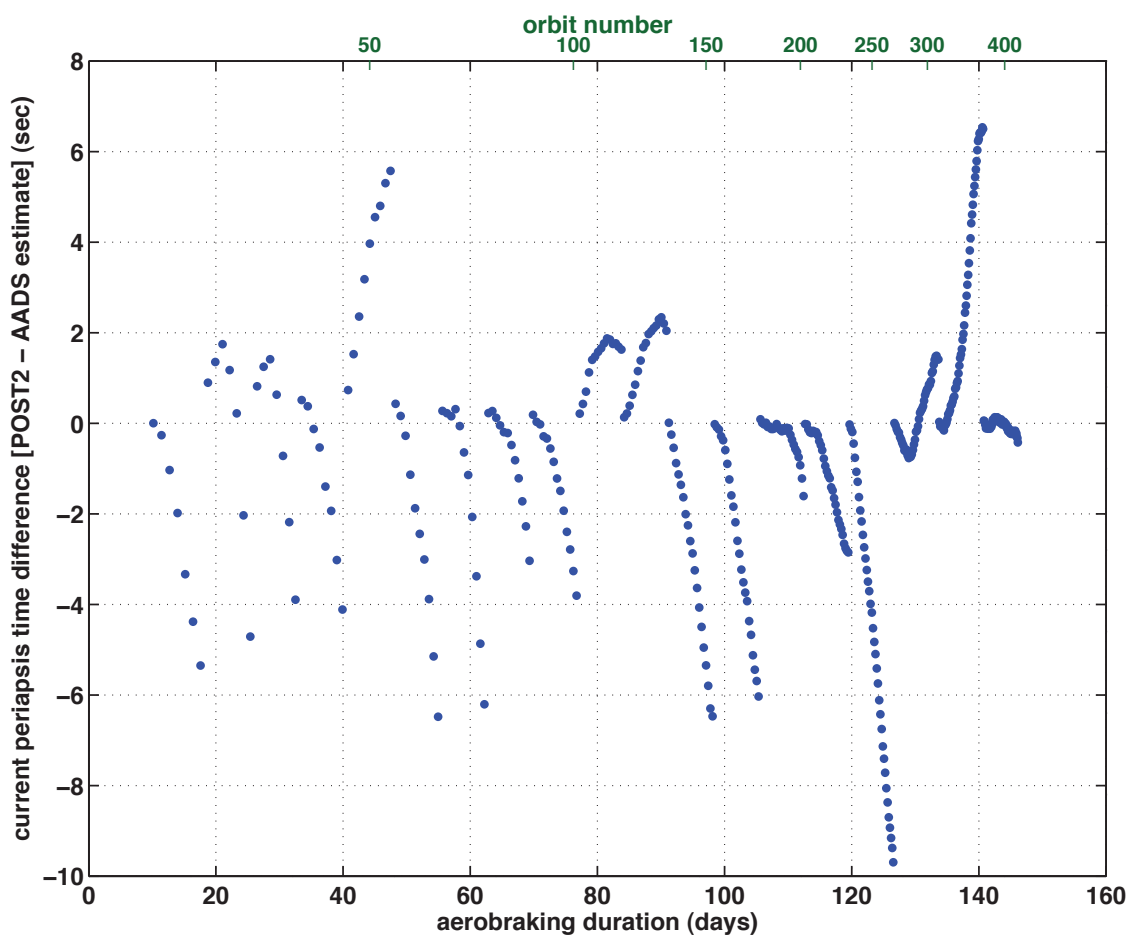

Figure 2. AADS Ephemeris Estimator Performance at Mars with Perturbed Atmosphere (and 7-day updates) using previous periapsis time predictions. 


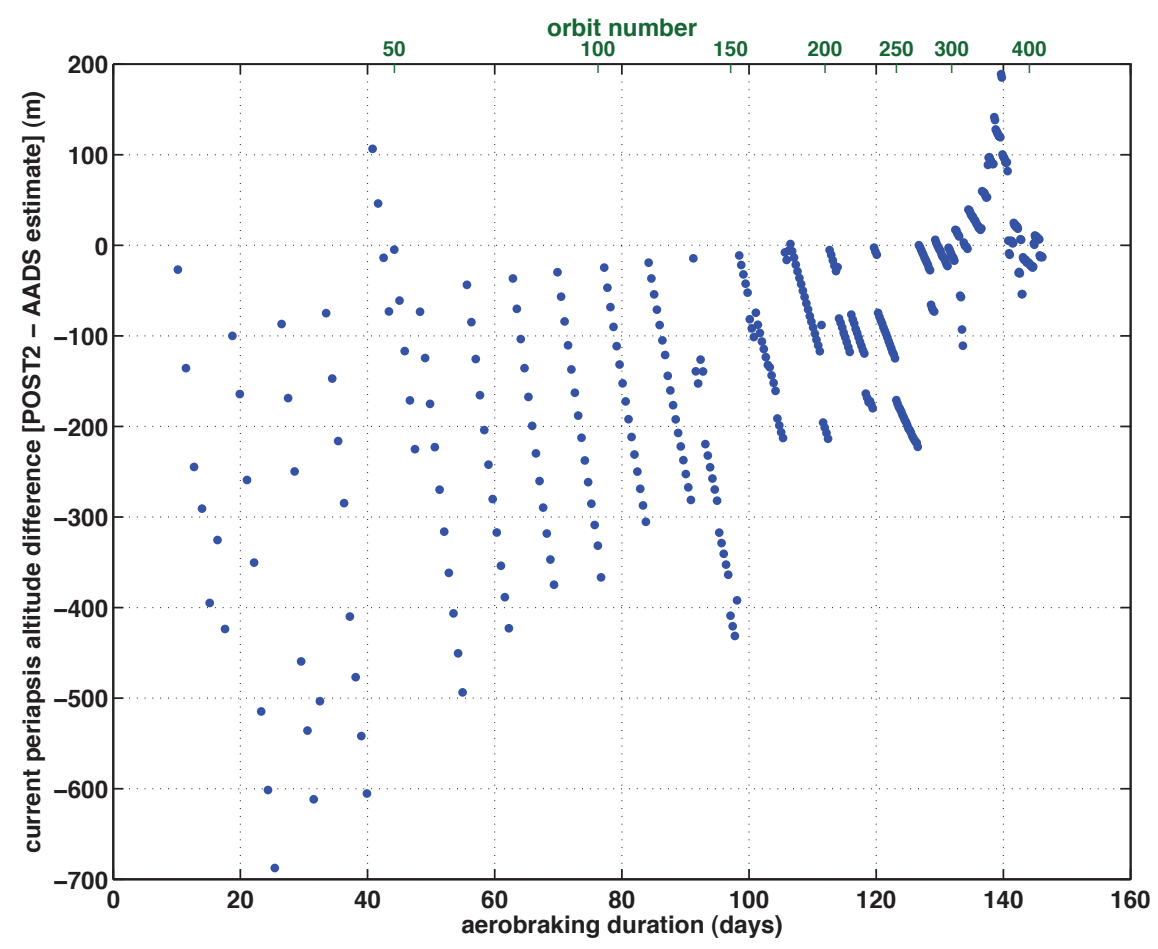

Figure 3. AADS Ephemeris Estimator Performance at Mars with Perturbed Atmosphere (and 7-day updates) using previous periapsis altitude predictions.

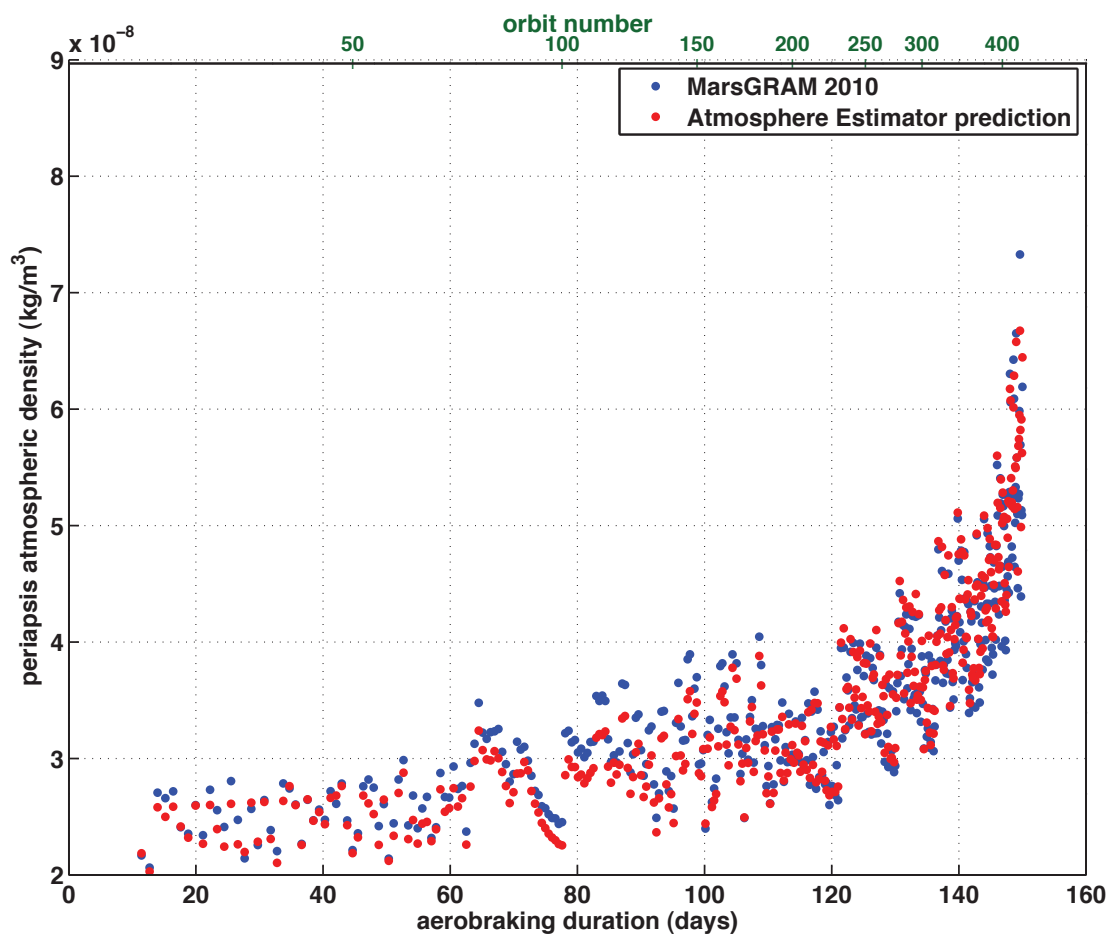

Figure 4. AADS Atmosphere Estimator Performance at Mars with Nominal Atmosphere (and 7-day updates) using predicted atmospheric density. 


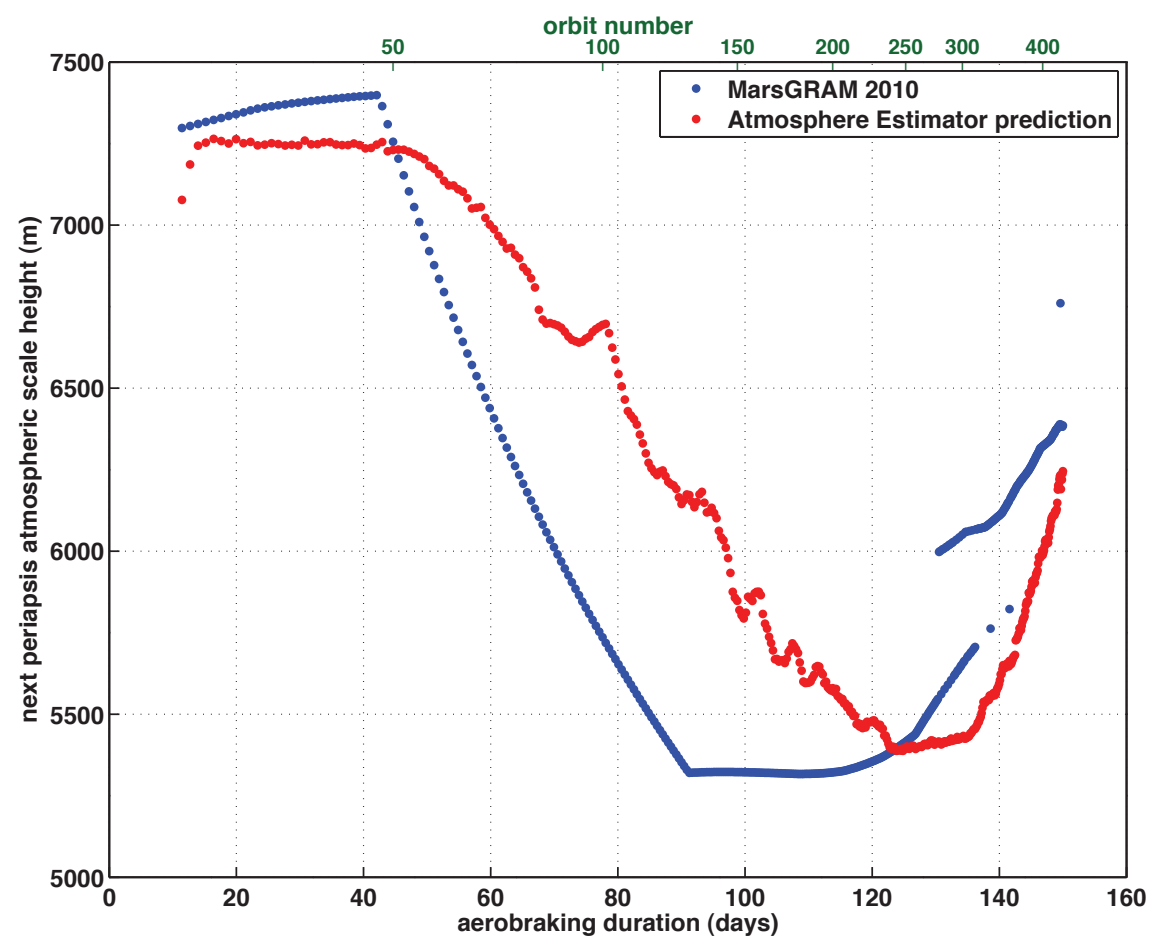

Figure 5. AADS Atmosphere Estimator Performance at Mars with Nominal Atmosphere (and 7-day updates) using predicted atmospheric scale height.

The Maneuver Estimator and AADS performance are highly coupled and can be assessed by determining how well the spacecraft remains within the desired operational corridor. At Mars, data from the Ephemeris and Atmosphere Estimators are used to estimate the freestream heat rate at periapsis during the next atmospheric pass. If the estimate is outside of the operational corridor, a maneuver is calculated such that the predicted heat rate at the next periapsis is at the target location within the corridor. First, a necessary change in altitude is calculated using Eq. (1):

$$
\Delta h=-H_{s} \cdot \ln \left(\frac{\rho_{\text {desired }}}{\rho_{\text {predicted }}}\right)=-H_{s} \cdot \ln \left(\frac{\dot{q}_{\text {desired }}}{\dot{q}_{\text {predicted }}}\right)
$$

where $\Delta h$ is the required altitude change, $H_{s}$ is the predicted atmospheric scale height, $\rho$ is the atmospheric density and $\dot{q}$ is the freestream heat rate. This change in altitude is added to the current estimated periapsis altitude, which is then added to the estimated apoapsis altitude to determine a new orbit semi-major axis, from which a new velocity at apoapsis is determined. The difference between this new apoapsis velocity and the current estimate of the apoapsis velocity is the required maneuver magnitude. This value is positive for a periapsis raise (decrease freestream heat rate) and negative for a periapsis lowering (increase freestream heat rate). The maneuver direction is estimated to be that of the pre-maneuver velocity vector at apoapsis. Since these maneuvers are typically small $(<0.5 \mathrm{~m} / \mathrm{s})$, this assumption works well, even when considering a finite burn.

\section{AADS at Mars and Comparison to Mission Runout}

With simulations complete for both the Mars aerobraking mission runout and AADS implementation, it is possible to compare the system performance between these two analyses. Figure 6 provides a comparison of the aerobraking mission profile of both the AADS (using a perturbed atmosphere and 7-day updates) and the mission runout simulation by using the difference between the aerobraking glide slope (orbit period versus time). Figure 7 shows the comparison based on the commanded maneuvers, Fig. 8 based on the orbit periapsis altitudes, and Fig. 9 based on periapsis locations (areocentric latitude) as a function of time. 


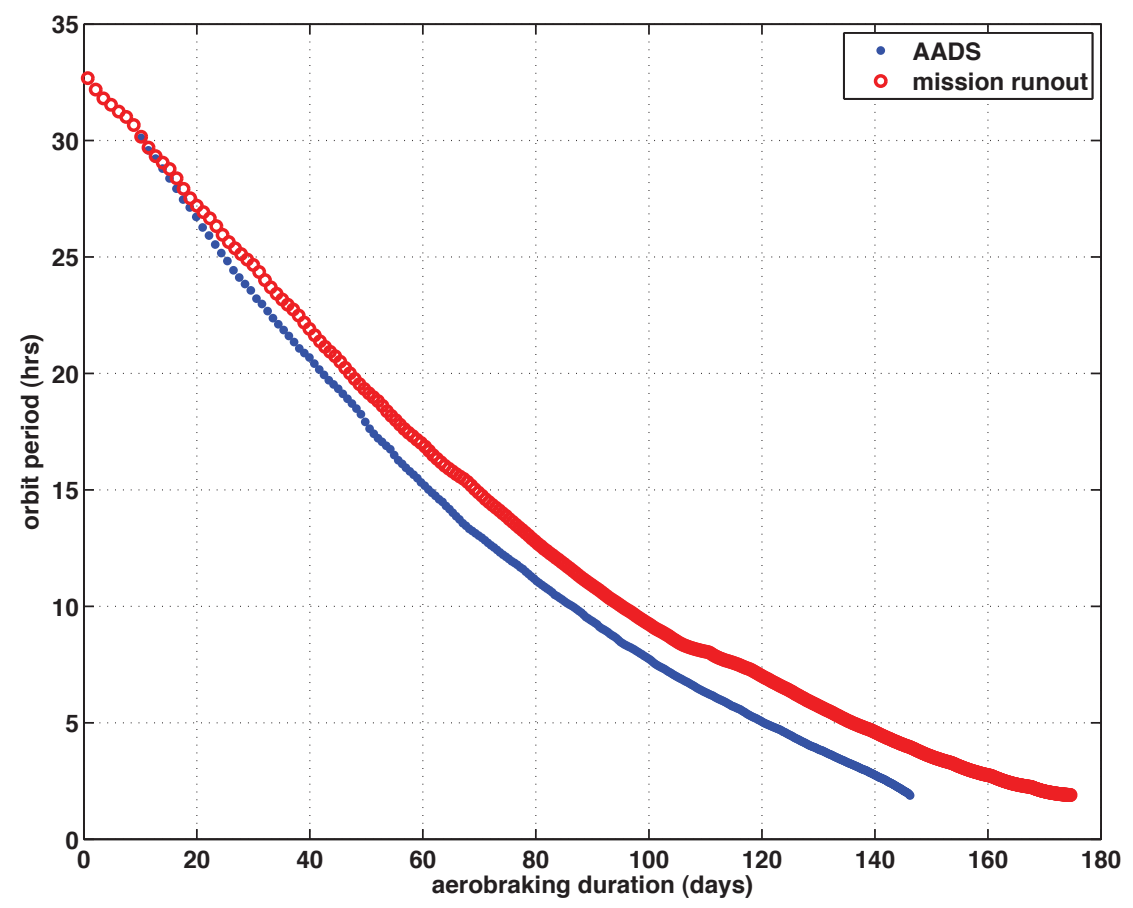

Figure 6. AADS with Perturbed Atmosphere (and 7-day updates) Comparison with Mission Runout at Mars using orbit period glide slope.

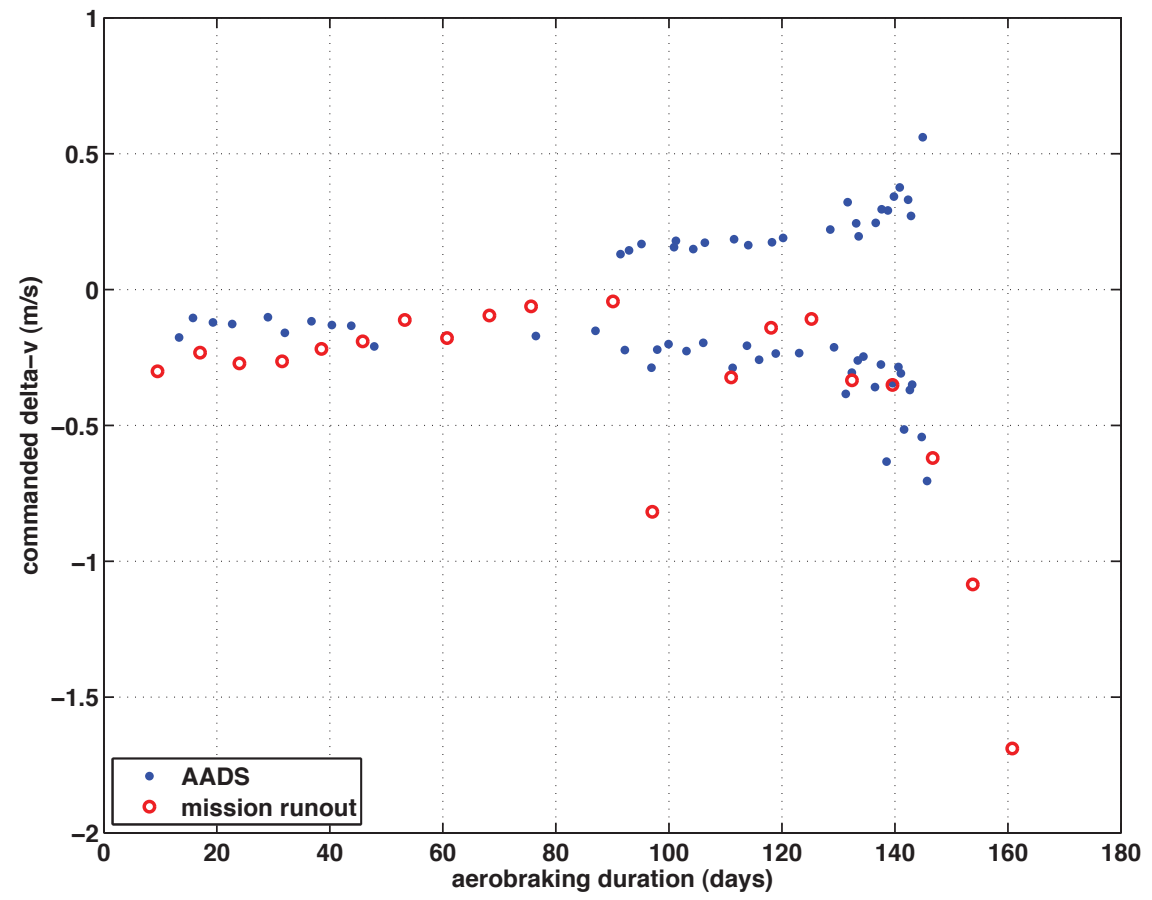

Figure 7. AADS with Perturbed Atmosphere (and 7-day updates) Comparison with Mission Runout at Mars using commanded maneuver $\Delta \mathbf{v}$. 


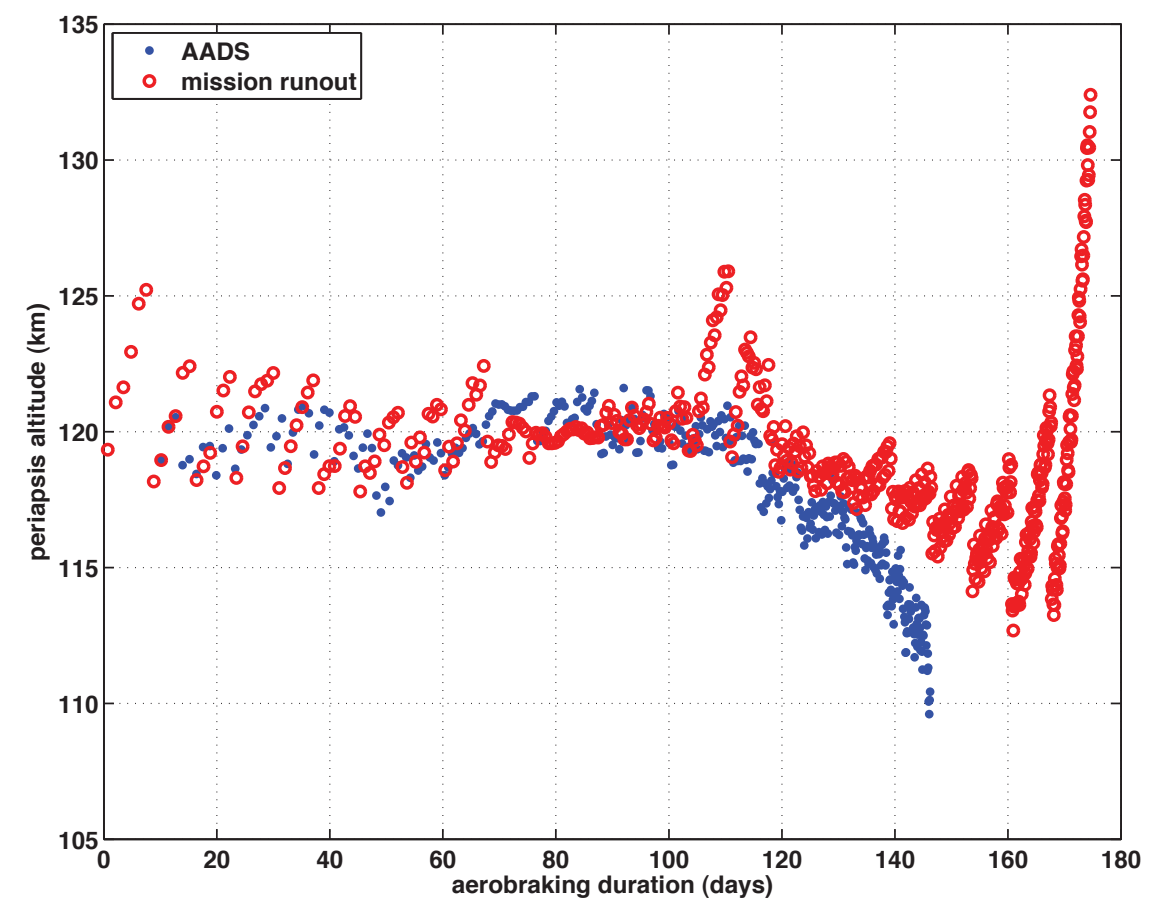

Figure 8. AADS with Perturbed Atmosphere (and 7-day updates) Comparison with Mission Runout at Mars using periapsis altitude.

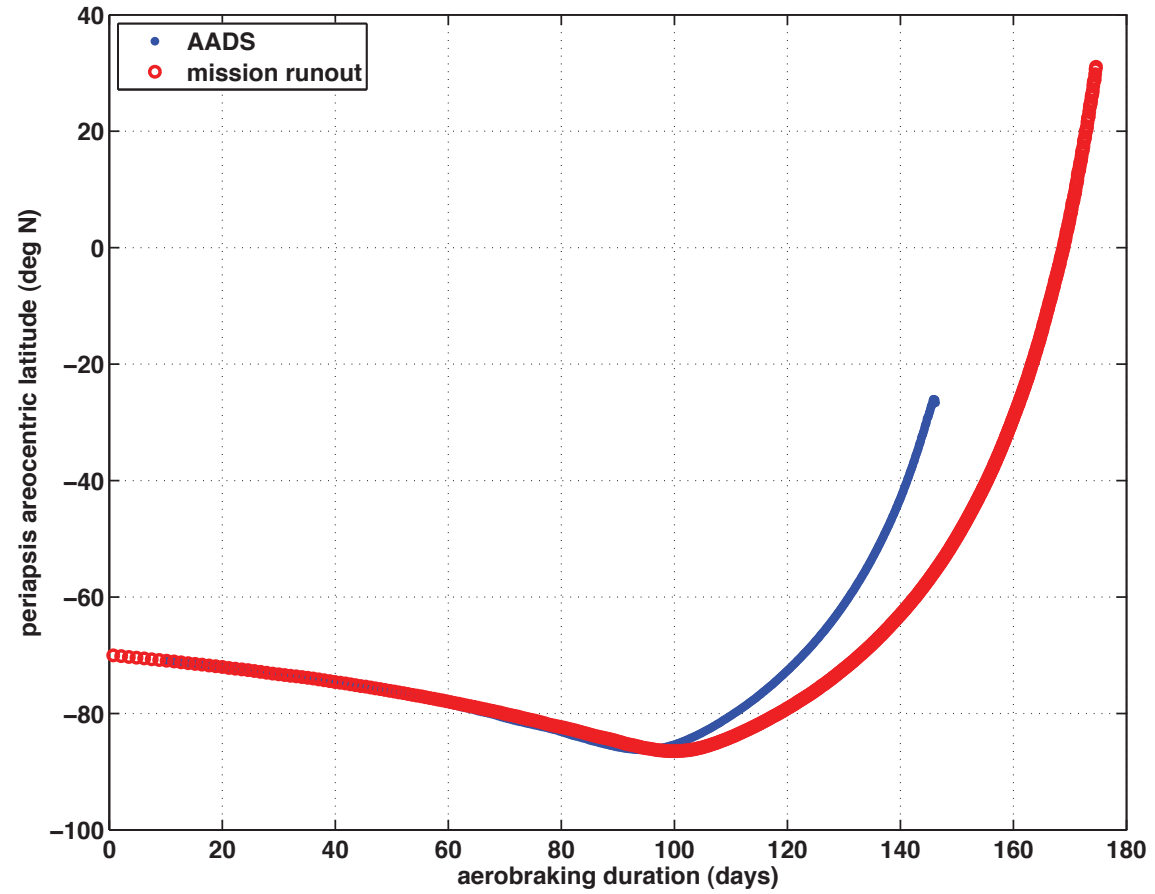

Figure 9. AADS with Perturbed Atmosphere (and 7-day updates) Comparison with Mission Runout at Mars using periapsis areocentric latitude. 
A summary of the AADS performance and margin for the Mars aerobraking mission simulation with a perturbed atmosphere and assuming 7 day ground updates, in terms of how well the spacecraft stays within the mission operations corridor, is provided in Fig. 10. This type of plot show that the AADS system is successful in keeping the spacecraft within the specified corridor, and also illustrates the difference between the AADS predicted freestream heat rate and the estimated actual heat rate, which is mainly driven by the differences between the Atmosphere Estimator density and scale height estimates from those of the MarsGRAM-2010 atmosphere model.

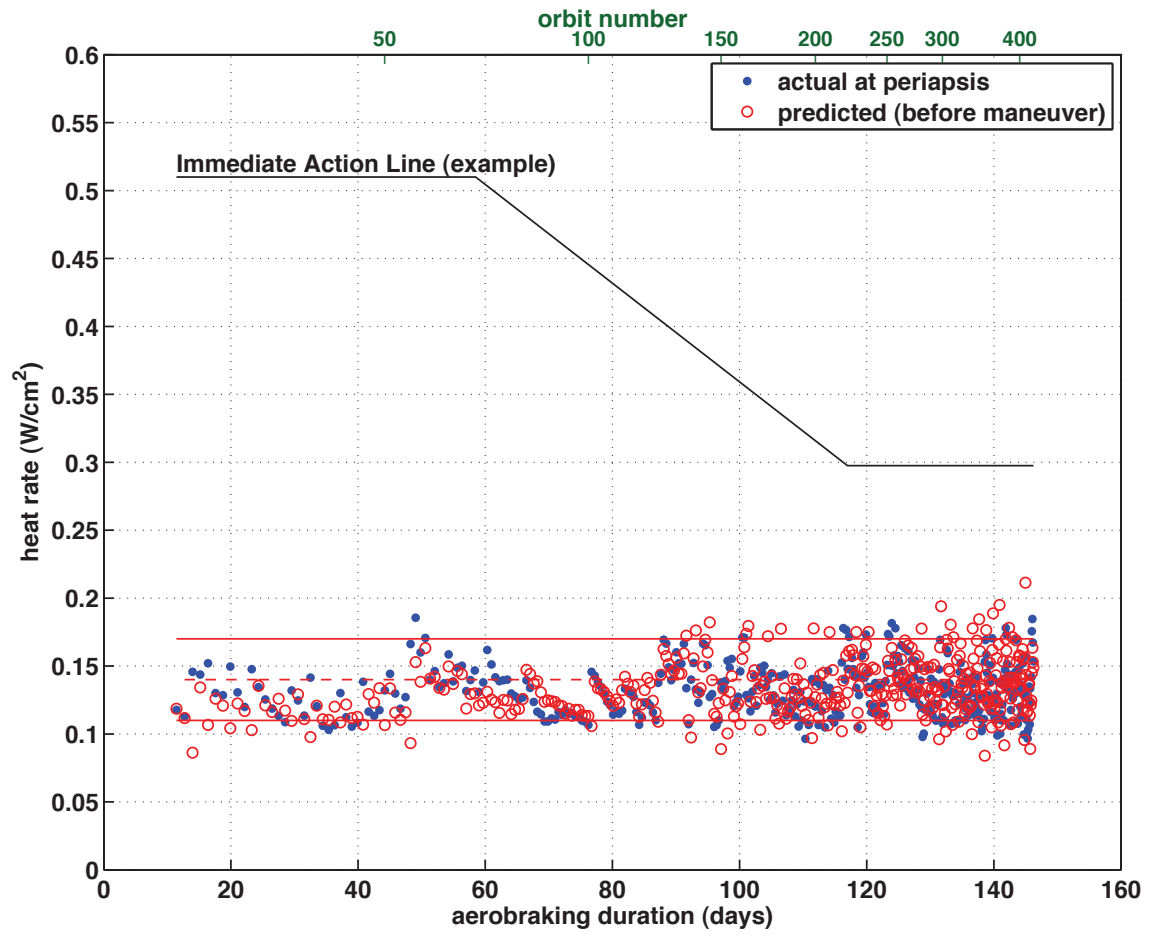

Figure 10. AADS Mission Operations Corridor Performance at Mars with a Perturbed Atmosphere and 7-day Updates.

Now that the AADS baseline performance analyses are complete for Mars and show successful operation with a perturbed atmosphere and the desired 7-day ground update cycle, it is possible to compare the AADS results against the mission runout analysis, which is summarized in Table 1.

The reduction in the aerobraking duration in the AADS simulation as compared to the mission runout is due to the AADS ability to perform a maneuver on each and every orbit, if necessary, whereas the mission runout only allows an orbit

Table 1. Summary of AADS Performance with a Perturbed Atmosphere (and 7-day updates) Compared to the Mission Runout at Mars

\begin{tabular}{|c|c|c|}
\cline { 2 - 3 } \multicolumn{1}{c|}{} & AADS & $\begin{array}{c}\text { Mission } \\
\text { Runout }\end{array}$ \\
\hline Aerobraking Duration (days) & 146.2 & 174.7 \\
\hline Total $\Delta \mathbf{v}(\mathrm{m} / \mathrm{s})$ & 15.9 & 7.9 \\
\hline Number of Maneuvers & 62 & 21 \\
\hline
\end{tabular}
once per week (as also illustrated in the number of maneuvers). The increase in total delta-v is mainly a consequence of comparing an AADS simulation which uses a perturbed atmosphere against the mission runout which uses a nominal atmosphere. These atmospheric perturbations have a strong effect on the AADS estimates, including the required maneuver magnitudes.

It should be noted that in these heat rate corridor plots, when the spacecraft "flies high in the corridor", it is actually dropping deep into Mars' atmosphere; likewise "flying low in the corridor" means the spacecraft is skimming higher in the atmosphere. It follows then that changing the corridor limits (and/or the target within the corridor) can have a significant effect on the aerobraking mission duration as well as the number of maneuvers performed. To illustrate this, the AADS aerobraking mission was simulated again using a tight operational corridor $\left(0.02 \mathrm{~W} / \mathrm{cm}^{2}\right.$ in width) centered at the nominal corridor lower limit $\left(0.11 \mathrm{~W} / \mathrm{cm}^{2}\right)$ and again at the nominal corridor 
upper limit $\left(0.17 \mathrm{~W} / \mathrm{cm}^{2}\right)$. Figures 11 and 12 illustrate the corridor performance for these cases and a summary of the mission performance is also provided in Table 2.

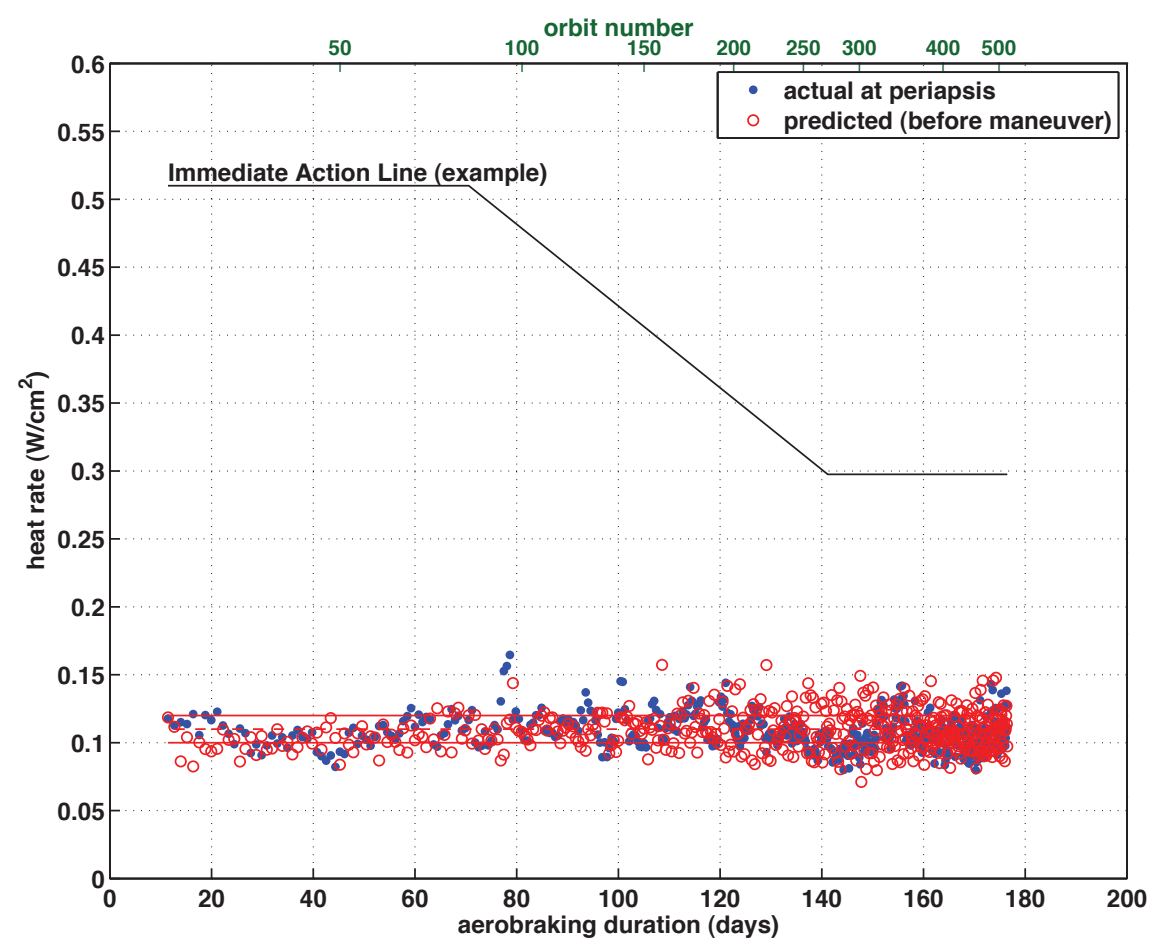

Figure 11. AADS Constrained Mission Operations Corridor Performance at Mars with a Perturbed Atmosphere (and 7-day updates) for a tight corridor centered on the nominal corridor lower limit.

Figure 12 does show some increased volatility in the AADS predictions as the aerobraking mission progresses for the more aggressive corridor, as compared to Fig. 11 which uses a more conservative corridor. This volatility is driven by the aggressive nature of the corridor (e.g. low altitude = higher density) and its impact on the accuracy of the Ephemeris Estimator's integration of the atmospheric pass IMU acceleration data. Since density information is estimated almost directly from acceleration data, and AADS will be more sensitive to these estimates due to the higher density environment, lack of sufficient resolution of the acceleration data will result in earlier and/or more rapid divergence within the AADS Ephemeris Estimator. Also, as the mission progresses and the orbit period reduces, the Ephemeris Estimator propagation relies more heavily on acceleration data as the atmospheric passes become longer and more frequent.

The Ephemeris Estimator's integration of the maneuver acceleration data also plays a small role in this behavior. Small maneuvers $(<0.5 \mathrm{~m} / \mathrm{s}, \sim 1 \mathrm{sec}$ burn duration) coupled with the fixed resolution of the acceleration data (10 $\mathrm{Hz}$ ) means that in many cases, the acceleration data may not capture the entire maneuver, thus resulting in poor Ephemeris Estimators estimates of the maneuver delta-v. Early in the aerobraking mission, when maneuvers are relatively sparse, this type of error is not very pronounced. Later in the mission, however, when maneuvers are much more frequent, recalling that they may occur on every orbit if necessary, the effects of this error in the delta-v estimate can quickly build up.
Table 2. Summary of AADS performance at Mars with a perturbed atmosphere for runs using the nominal operational corridor, a corridor constrained to the nominal corridor upper limit, and one to the nominal corridor lower limit.

\begin{tabular}{|c|c|c|c|}
\cline { 2 - 4 } \multicolumn{1}{c|}{} & $\begin{array}{c}\text { Nominal } \\
\text { Corridor }\end{array}$ & $\begin{array}{c}\text { Corridor at } \\
\text { Lower Limit }\end{array}$ & $\begin{array}{c}\text { Corridor at } \\
\text { Upper Limit }\end{array}$ \\
\hline Aerobraking Duration (days) & 146.2 & 176.5 & 116.4 \\
\hline Total $\Delta \mathbf{v}(\mathrm{m} / \mathrm{s})$ & 15.9 & 36.6 & 28.9 \\
\hline Number of maneuvers & 62 & 249 & 227 \\
\hline
\end{tabular}




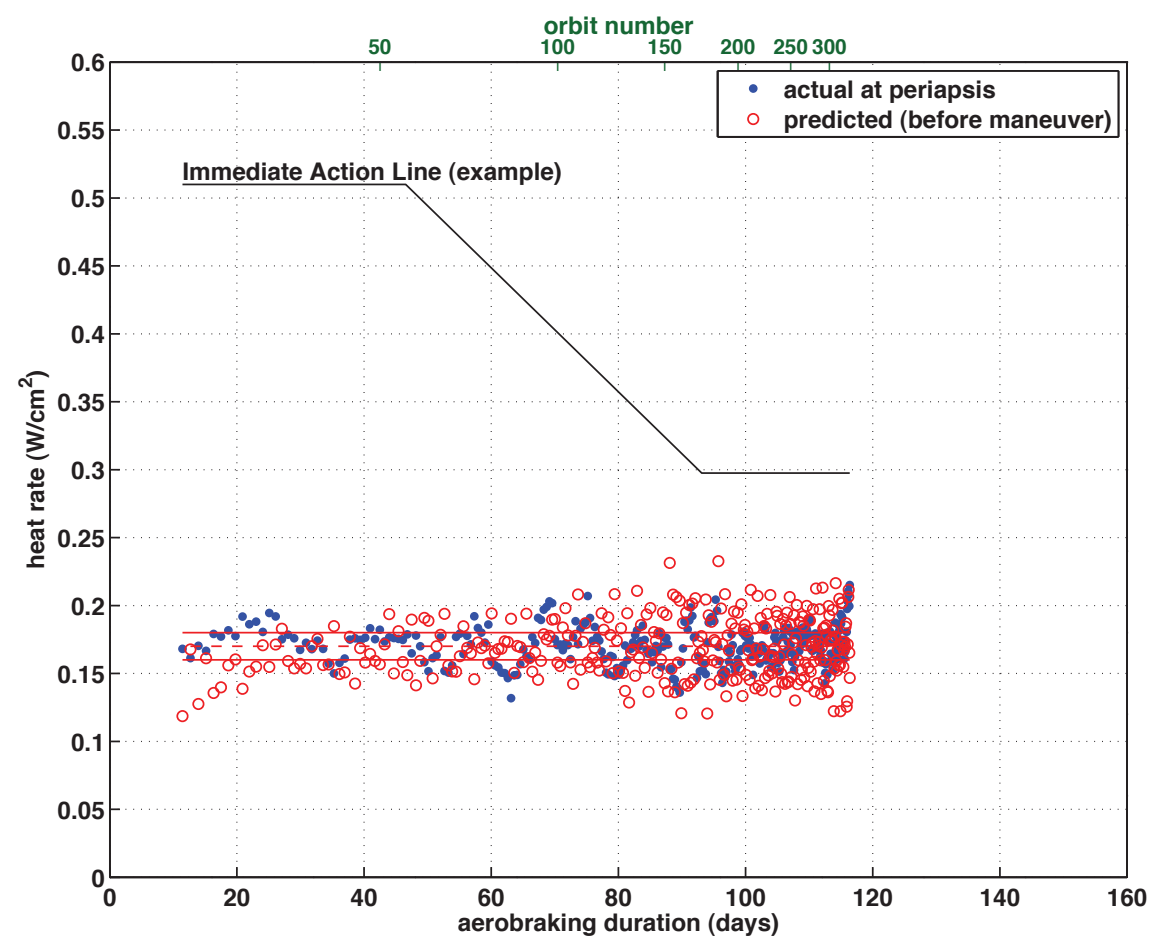

Figure 12. AADS Constrained Mission Operations Corridor Performance at Mars with a Perturbed Atmosphere (and 7-day updates) for a tight corridor centered on the nominal corridor upper limit.

The accuracy at which AADS can maintain such a tight operational corridor will also be driven by the accuracy in the predicted freestream heat rate. Any improvements in the Atmosphere Estimator density and scale height estimates will narrow the spread between the predicted and actual, improve the required maneuver estimate to reach the target in the corridor, and thus further improve the overall corridor performance.

This effect of corridor selection on mission duration can be further emphasized by evaluating the AADS mission performance for a corridor with the same width as the nominal analysis $\left(0.06 \mathrm{~W} / \mathrm{cm}^{2}\right)$, but shifted up by that same amount $\left(0.17-0.23 \mathrm{~W} / \mathrm{cm}^{2}\right)$ in order to significantly reduce the time required for aerobraking, as shown in Fig. 13. Table 3 provides a comparison between the nominal and this more aggressive aerobraking mission, illustrating that there is no significant impact to the number of maneuvers and/or delta-v required (which are driven more by the corridor size and target). Figure 13 also shows the same

Table 3. Comparison of AADS Performance at Mars with a Perturbed Atmosphere (and 7-day updates) for the Nominal and Aggressive Operational Corridors

\begin{tabular}{|c|c|c|}
\cline { 2 - 3 } \multicolumn{1}{c|}{} & $\begin{array}{c}\text { Nominal } \\
\text { Corridor }\end{array}$ & $\begin{array}{c}\text { Aggressive } \\
\text { Corridor }\end{array}$ \\
\hline Aerobraking Duration (days) & 146.2 & 101.1 \\
\hline Total $\Delta \mathrm{v}(\mathrm{m} / \mathrm{s})$ & 15.9 & 13.9 \\
\hline Number of Maneuvers & 62 & 69 \\
\hline
\end{tabular}
immediate action line limits as for the nominal mission corridor, further illustrating the importance of selecting an operational corridor which ensures sufficient spacecraft safety margin throughout the aerobraking mission.

An additional analysis which expands upon this idea of aerobraking mission timeline control, and also demonstrates how AADS can be used to reduce risk during off-nominal aerobraking operations, was also completed using the AADS POST2 simulation at Mars. In this simulation, after some period of time in nominal aerobraking, AADS is instructed (through the spacecraft ground update interface) to dramatically lower the operational corridor. At Mars, this raises the periapsis altitude higher than would have been during the nominal mission, thus reducing drag, which results in the spacecraft "falling behind" the nominal glide slope. After approximately 1 week, AADS is commanded to increase the upper corridor limit back to where it was for the nominal mission, as well as dramatically increase the lower corridor limit. This decreases the periapsis altitude target deeper into the atmosphere, increasing drag, steepening the glide slope, and allowing the spacecraft to eventually recover the 
nominal glide slope after about 2 weeks. Figure 14 shows the effect of this scenario on the glide slope profile, as compared to the nominal mission, while Figures 15 and 16 show the effect on the operational corridor and resulting AADS performance.

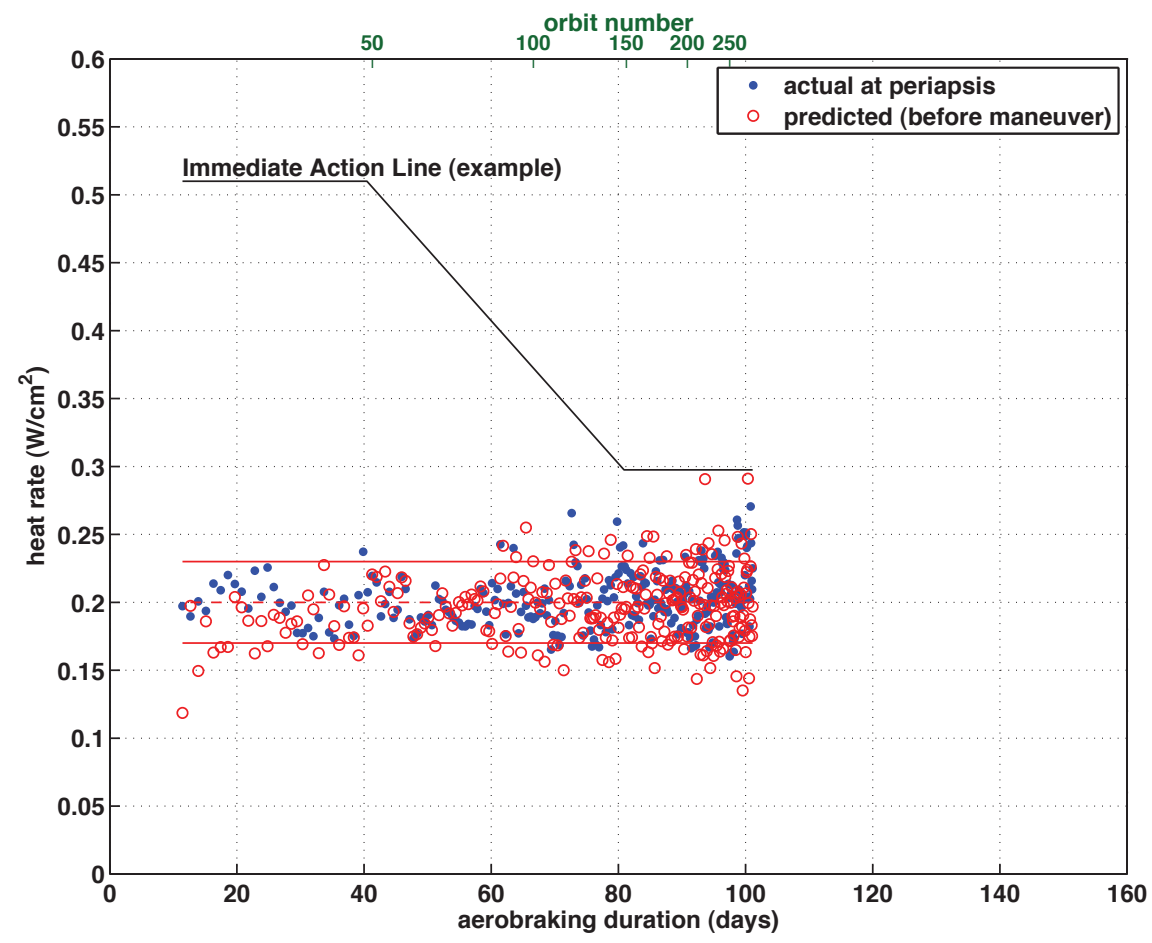

Figure 13. AADS Mission Aggressive Operations Corridor Performance at Mars with a Perturbed Atmosphere (and 7-day updates).

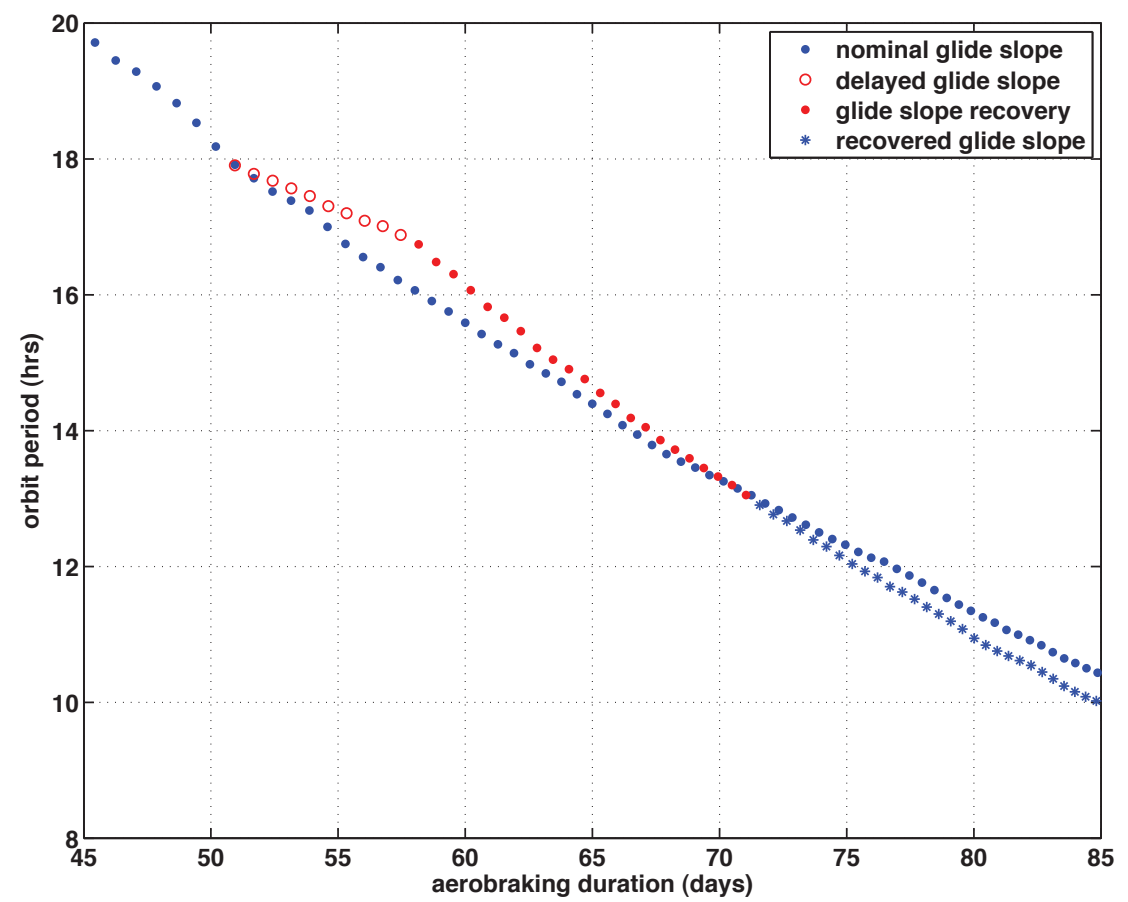

Figure 14. AADS Glide Slope Delay and Recovery Scenario at Mars with a Perturbed Atmosphere (and 7day updates). 


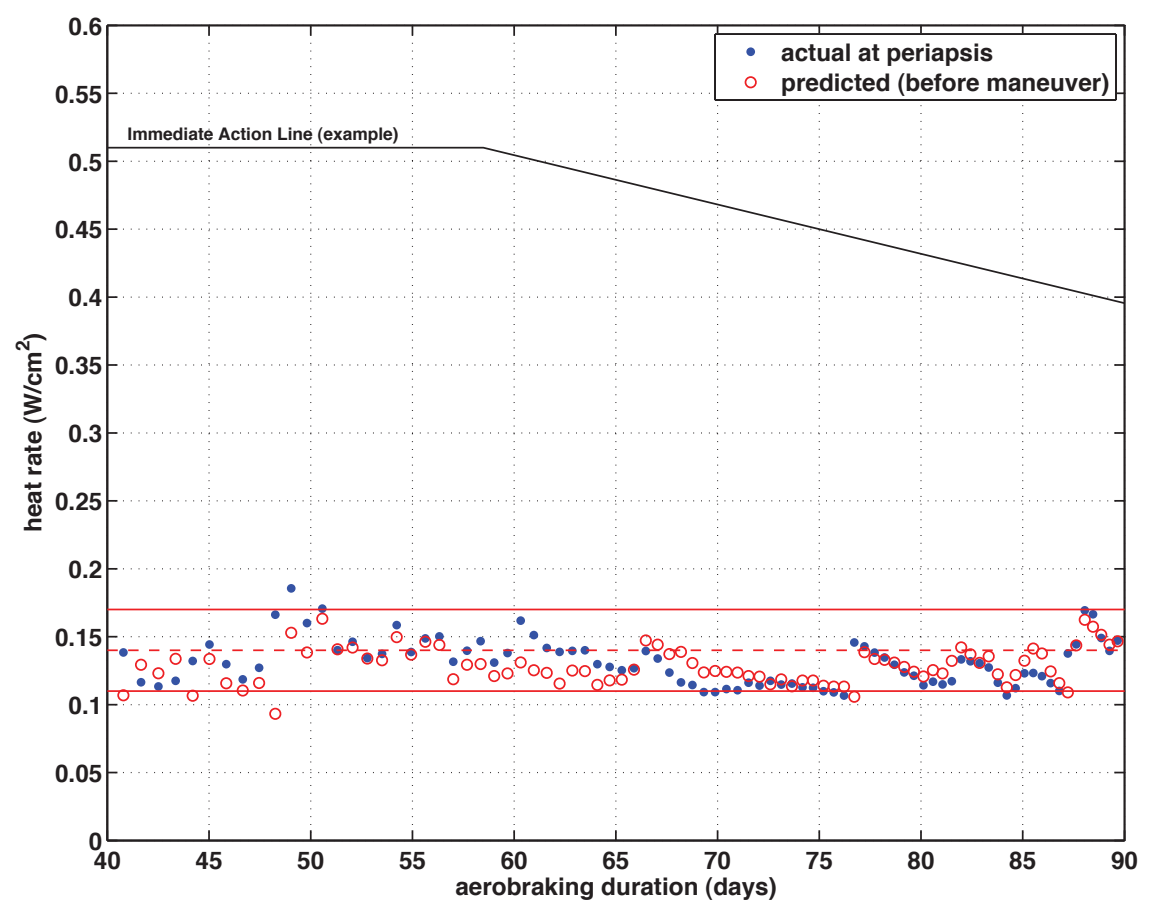

Figure 15. AADS Mission Operations Corridor Performance Snapshot for the nominal corridor.

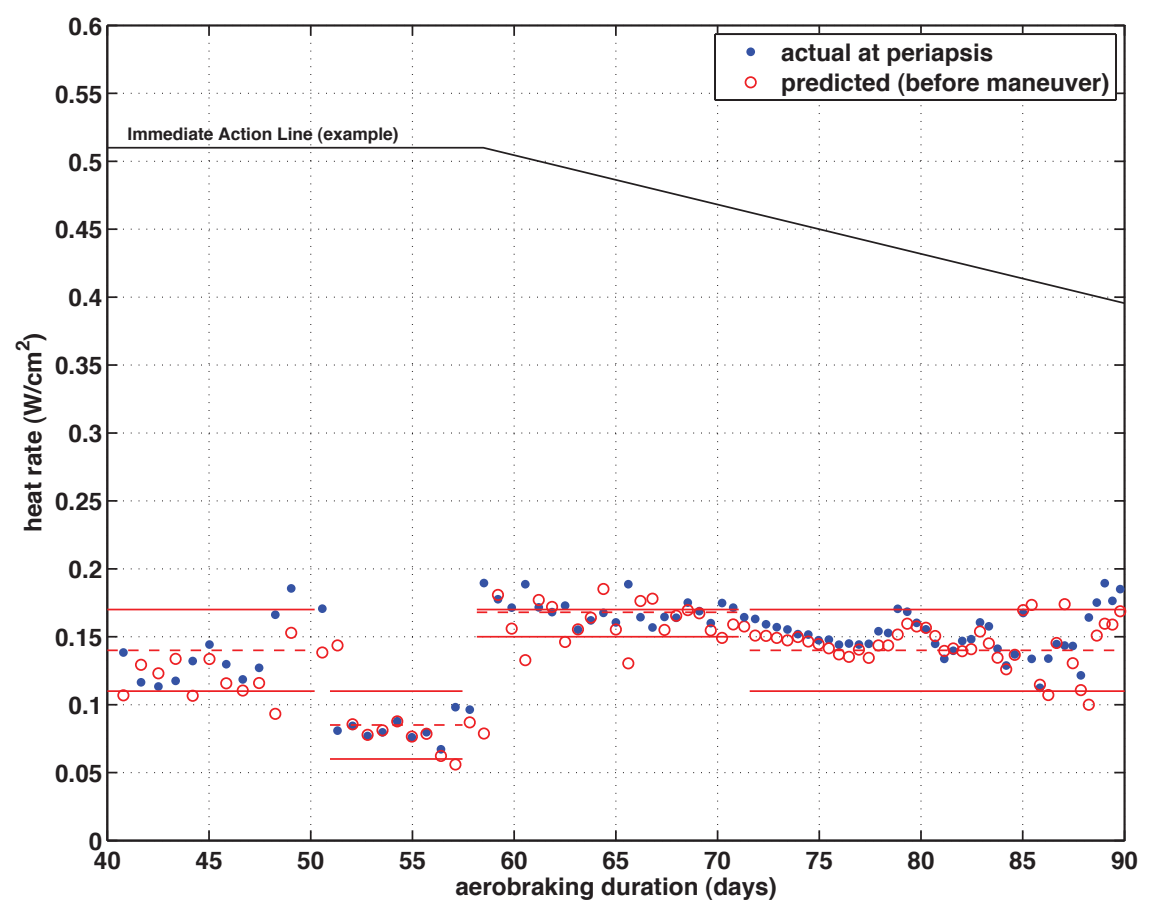

Figure 16. AADS Mission Operations Corridor Performance Snapshot for the glide slope delay and recovery corridor.

Note that in Fig. 14, it shows that not only is the nominal mission glide slope recovered, but the glide slope is actually slightly steeper. This is a result of the spacecraft being left high in the corridor at the end of the recovery period, much higher than during the same time period in the nominal case (see Figs. 15 and 16). The natural tendency is for the spacecraft to remain near the top of the corridor for over 2 weeks before the first maneuver is required. If it is deemed necessary to immediately return closer to the nominal glide slope, this could simply be 
achieved by lowering the top of the corridor (which actually increases spacecraft safety margin), resulting in AADS to command a maneuver to push the spacecraft lower into the corridor, until the nominal glide slope is fully recovered. At that time, the corridor upper limit can be returned to its nominal value.

With AADS, this scenario is successfully achieved autonomously, with only 3 required updates from the spacecraft: (1) delay the glide slope, (2) recover the glide slope, and (3) return to nominal glide slope, and is accomplished simply by changing the desired corridor and target, thus altering when and how many maneuvers are executed during the affected time period. In addition, it is significant to note that all of this is accomplished without the need to violate the nominal mission upper corridor limit, thus introducing no additional spacecraft risk. With ground based aerobraking operations, recovery of the nominal glide slope would likely not be possible without increasing the upper corridor limit due to the constraint on maneuver execution frequency.

\section{AADS at Venus and Comparison to the Mission Runout}

At Venus, data from the Ephemeris and Atmosphere Estimators are used with a spacecraft thermal model (Ref. 13) to estimate the spacecraft (solar panel) temperature at periapsis during the next atmospheric pass. If the predicted temperature lies outside of the operational temperature corridor, the same spacecraft thermal model is used to estimate the desired atmospheric density, given the predicted spacecraft orbital state at the next periapsis, and the desired target temperature within the corridor. From this information, a maneuver is calculated based on the necessary change in altitude, calculated using Eq. (2):

$$
\Delta h=-H_{s} \cdot \ln \left(\frac{\rho_{\text {desired }}}{\rho_{\text {predicted }}}\right)
$$

where $\Delta h$ is the required altitude change, $H s$ is the predicted atmospheric scale height, and $\rho$ is the atmospheric density. This change in altitude is added to the current estimated periapsis altitude, which is then added to the estimated apoapsis altitude to determine a new orbit semi-major axis, from which a new velocity at apoapsis is determined. The difference between this new apoapsis velocity and the current estimate of the apoapsis velocity is the required maneuver magnitude. As with Mars, this value is positive for a periapsis raise (decrease spacecraft temperature) and negative for a periapsis lowering (increase spacecraft temperature), and the maneuver direction is estimated to be that of the pre-maneuver velocity vector at apoapsis.

Many other differences exist between aerobraking missions at Mars and at Venus which affect the performance of the AADS. For example, at Venus, the effects of the Sun, both in terms of 3rd body gravitational effects and solar radiation pressure, have a real influence on the spacecraft orbit. This, coupled with the higher orbital velocities (due to the more massive planet), and the much higher atmospheric density, all contribute to what would likely drive a mission operations team to utilize a temperature corridor during aerobraking, as opposed to the heat rate corridor used at Mars. In addition, the atmospheric perturbations at Venus (as provided by VenusGRAM) are much less than those at Mars. This means that it would be expected that at Venus, as is the case at Mars, an update frequency up to 7 days, or longer, will provide sufficient AADS performance to insure spacecraft safety throughout the aerobraking mission.

A summary of the AADS performance within the mission operations temperature corridor for the Venus aerobraking mission simulation with a perturbed atmosphere (and 7-day updates) is provided in Figure 17.

The strong solar effects are clearly visible where the spacecraft is pulled to either the top of the corridor or to the bottom, depending on where the Sun is located relative to Venus and the spacecraft. In order to better take advantage of these effects, the target within the corridor can be varied during the mission using the weekly update capability, as illustrated in Figure 18. This approach can reduce the number of maneuvers required to maintain the operational corridor without the need to change the corridor itself.

Table 4 provides a summary comparing the Venus AADS baseline performance, using a perturbed atmosphere and 7-day updates, for both the fixed and varying corridor target, with the mission runout analysis. The difference in the duration between the AADS and mission runout can be attributed to the slight differences in the (nominal) corridor target. The reduction in the number of maneuvers by utilizing a variable target is also clearly evident.

\section{Table 4. Summary of AADS Performance with Perturbed Atmosphere (and 7-day updates) Compared to Mission Runout at Venus.}

\begin{tabular}{|c|c|c|c|}
\cline { 2 - 4 } \multicolumn{1}{c|}{} & $\begin{array}{c}\text { AADS } w / \\
\text { Fixed Target }\end{array}$ & $\begin{array}{c}\text { AADS w/ } \\
\text { Variable Target }\end{array}$ & $\begin{array}{c}\text { Mission } \\
\text { Runout }\end{array}$ \\
\hline Aerobraking Duration (days) & 146.2 & 176.5 & 116.4 \\
\hline Total $\Delta \mathrm{v}(\mathrm{m} / \mathrm{s})$ & 15.9 & 36.6 & 28.9 \\
\hline Number of maneuvers & 62 & 249 & 227 \\
\hline
\end{tabular}




\section{AADS at Titan and Comparison to Mission Runout}

At Titan, data from the Ephemeris Estimator is used to estimate the spacecraft altitude at periapsis during the next atmospheric pass. If the predicted altitude is outside of the operational altitude corridor, the difference between the predicted and the desired target altitude within the corridor is used to calculate the required correction maneuver. This change in altitude is then added to the current estimated periapsis altitude, which is then added to the estimated apoapsis altitude to determine a new orbit semi-major axis, from which a new velocity at apoapsis is determined. The difference between this new apoapsis velocity and the current estimate of the apoapsis velocity is the required maneuver magnitude. As with Mars and Venus, this value is positive for a periapsis raise (increase in altitude) and negative for a periapsis lowering (decrease in altitude), and the maneuver direction is estimated to be that of the premaneuver velocity vector at apoapsis.

To even a greater extent than was the case for Venus, aerobraking at Titan needs to account for strong 3rd body effects, but in this case, from Saturn. These gravitational effects can once again be utilized in selection of the corridor target in order to minimize the number of maneuvers required to maintain spacecraft safety within the corridor. Also, as was the case with Venus, the atmospheric perturbations provided from TitanGRAM are very small compared to those from MarsGRAM (and are even smaller than those from VenusGRAM as well). In addition, since this Titan analysis makes use of an altitude corridor, atmospheric perturbations (in density), in conjunction with the large atmospheric scale height, have little to no effect on the spacecraft location within the corridor itself. Therefore, as is the case with Mars and Venus, an update frequency up to 7 days, or longer, will provide sufficient AADS performance to insure spacecraft safety throughout the aerobraking mission.

A summary of the AADS performance for the Titan aerobraking mission simulation with a perturbed atmosphere (and 7-day updates) is provided in Fig. 19. Table 5 provides a summary comparing the AADS mission simulation with the mission runout analysis.

Table 5. Summary of AADS Performance with a Perturbed Atmosphere (and 7-day updates) Compared to the Mission Runout at Titan

\begin{tabular}{|c|c|c|}
\cline { 2 - 3 } \multicolumn{1}{c|}{} & AADS & $\begin{array}{c}\text { Mission } \\
\text { Runout }\end{array}$ \\
\hline Aerobraking Duration (days) & 75.5 & 74.1 \\
\hline Total $\Delta \mathbf{v}(\mathrm{m} / \mathrm{s})$ & 105.0 & 105.4 \\
\hline Number of Maneuvers & 9 & 9 \\
\hline
\end{tabular}

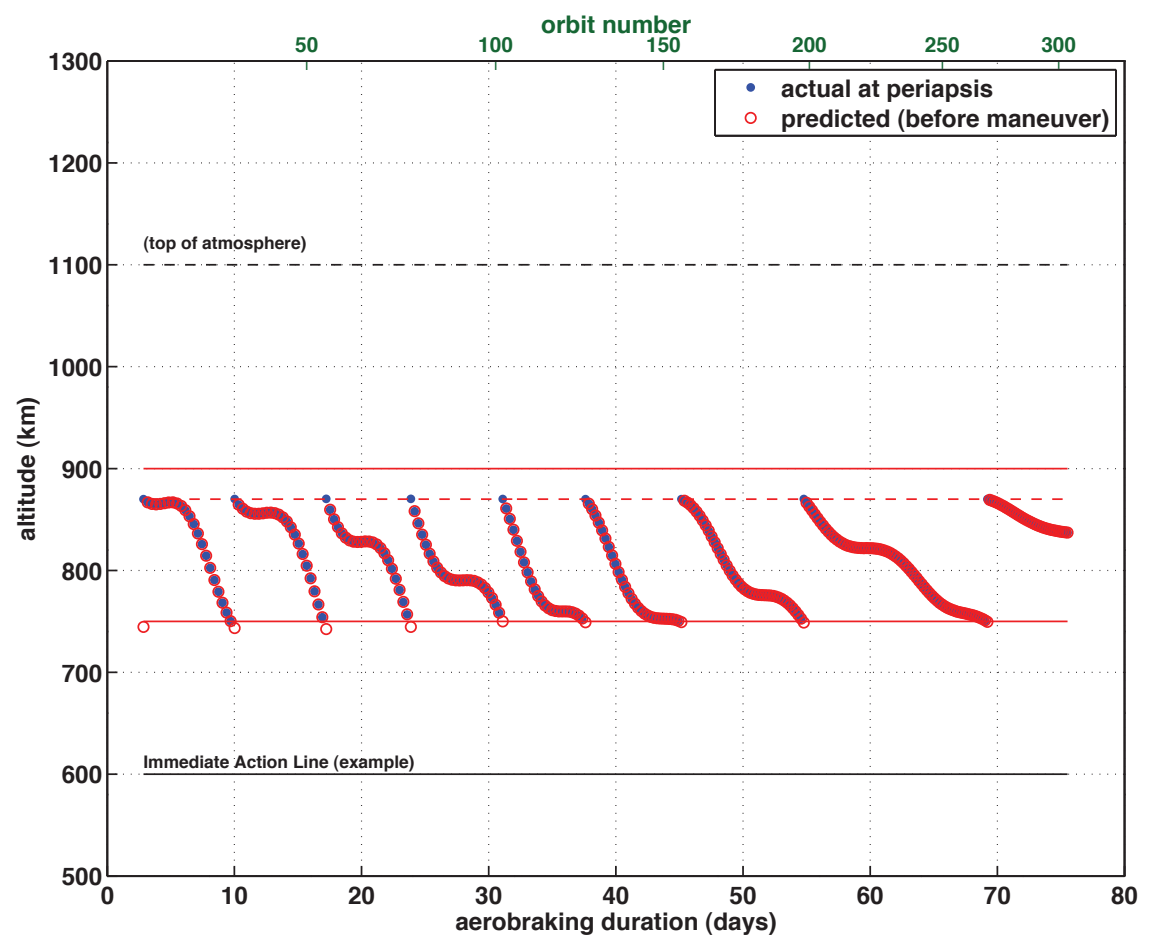

Figure 19. AADS Mission Operations Corridor Performance at Titan with a Perturbed Atmosphere (and 7-day updates). 


\section{AADS Phase 2 Development and Status}

\section{A. AADS Simulation Model Development}

At the start of Phase 2, work has been focused on updates to the various AADS modules in order to both improve performance and prepare for the next level more detailed analysis.

\section{Ephemeris Estimator}

A new Ephemeris Estimator has been developed for the AADS in Phase 2 analyses. This new State Propagator is designed with an architecture much better suited for onboard spacecraft applications. It is divided into two main functions: State Determination and Orbital Propagation. The State Determination function is a low rate, real-time state determination function, which maintains the current spacecraft state estimate while making use of all available IMU acceleration data. The Orbit Propagation function executes as a batch process, once per orbit, similar to the Phase 1 Ephemeris Estimator, to predict forward in time to estimate spacecraft state information.

The design of the State Propagator, particularly in the selection of the integration method, is likely to provide much improved state determination and state estimation, reducing both position and timing errors in the prediction of the next periapsis event, which is critical in helping improve the performance of the Atmosphere Estimator.

\section{Atmosphere and Maneuver Estimators}

In Phase 2, the Atmosphere Estimator development has focused on providing estimates on the uncertainties associated with the provided density estimates. This information is critical to the AADS (and the spacecraft) in understanding the scale of the uncertainty in the estimates of the next periapsis location with respect to the operational corridor. With this information, additional logic and safety triggers will be added to the Maneuver Estimator to react appropriately (i.e. bias the next maneuver, or even execute a "pop-up" maneuver, if necessary) if the density uncertainty is sufficiently large such that it indicates the spacecraft may exceed operational safety constraints (i.e. immediate action line).

\section{POST2 "Truth" Simulation}

Since the end of Phase 1, a new version of the POST2 simulation software was made available to the AADS team. This new software included conversion of the core code from FORTRAN to C, as well as some architecture changes to improve runtime performance. The AADS simulation was integrated with this new POST2 simulation (which required NO changes to the AADS software itself), and Phase 1 analyses were re-run to confirm the execution and performance of the AADS was unaffected.

Phase 2 will also provide an opportunity for analysis of the AADS system in a 6-DoF simulation, as compared to the 3-DoF analyses performed in Phase 1. Additional spacecraft models (including performance error, noise, uncertainties, etc.) are being added to the POST2 simulation, including sensors as an IMU and a startracker, as well as other spacecraft models such as thrusters and reaction wheels, while also including the complete set of mass properties (i.e. moments and products of inertias) of the representative MRO spacecraft.

There may also be the opportunity in Phase 2 to include in the "truth" simulation a reconstructed Mars atmosphere, based on flight data from the Mars Odyssey and MRO missions. This type of data would provide additional orbit-to-orbit variations than what is included in the MarsGRAM atmosphere model. Demonstrating that the AADS can still perform as desired under these more "flight-like" conditions is critical to ensuring the robustness of the AADS system.

\section{B. AADS Analyses}

A great deal of analyses are planned for Phase 2 of the AADS development. The first level of analyses will simply be a repeat of several of the Phase 1 analyses to ensure that the AADS system performance is maintained, if not improved, due to the many model updates previously described, primarily the replacement of the Ephemeris Estimator with the new State Propagator. Also, testing of the new Atmosphere Estimator density prediction uncertainties and the new Maneuver Estimator logic based on these uncertainties will be performed.

The next level of analyses will focus on determining the robustness of the AADS system. These analyses will utilize sensitivity and stress testing, in the form of Monte Carlo analyses, dispersing (to the appropriate level) the environment, spacecraft sensor and other spacecraft models and understanding the effects of these on the AADS performance.

The final level of analyses will be directed as a "fly-off" between the conventional ground-based aerobraking operations approach and utilization of the AADS. This will be done in an Operational Readiness Test (ORT) -like 
setting where a short segment (i.e. 1-2 weeks) of the mission operations can be simulated over the course of a few days. This same mission segment will be simulated using the current daily operations approach, including the size of the operations team, the amount of analysis required, a running account of expect DSN coverage, etc., both in nominal and off-nominal conditions, and the performance (i.e. how well the spacecraft maintains the operational corridor and/or the mission specified glide slope) will be compared to the same simulation using the weekly AADS operations approach. Mission performance, and likely even more importantly, an accounting of the resources required for each operations approach can then be easily compared side-by-side. This will be the true test as to whether the AADS is providing the desired performance and operational advantages.

\section{Conclusion}

The purpose of Phase 1 of the NESC AADS study was to provide an assessment of the feasibility in development of the models and tools capable of autonomous aerobraking. During Phase 1, atmospheric, aerodynamic, and thermal models for a representative spacecraft, MRO, were developed for both the onboard AADS, and a ground-based "truth" simulation in POST2, developed for testing purposes. This was to provide a proof-of-concept that these models and tools can be put together in such a way as to provide a means to move much of the aerobraking daily operations activities onboard a spacecraft.

For Phase 1, AADS was tested in simulated aerobraking missions at three destinations, each requiring a unique aerobraking corridor type. At Mars, the corridor was based on a freestream heat rate indicator. Due to its proximity to the Sun, the aerobraking mission at Venus utilized a spacecraft (solar panel) temperature corridor. Finally an altitude corridor was used to at Titan. Results of the Phase 1 analysis show that an autonomous aerobraking system can be developed and tested in a high fidelity simulation environment, indicating it is quite feasible that a spacecraft that wishes to utilize aerobraking (at any of these destinations) can do so safely with no more than one ground update every 7 days. The Phase 1 analyses also demonstrates the added flexibility of such a system in terms of corridor and/or glide slope control (as well as other desired constraints) as well as the ability to quickly recovery from an off-nominal event, due to this added flexibility, something the conventional aerobraking operations approach does not offer.

Phase 2 of the AADS development will provide the next step to implementation of the autonomous aerobraking software onboard a spacecraft. Increased fidelity in both the AADS modeling, as well as the "truth" simulation itself, will provide for an opportunity to show the robustness of the AADS system in light of the expected uncertainties of mission and spacecraft operations. Ultimately, Phase 2 hopes to show, in an operational test scenario, the desired performance and operational resource advantages over conventional aerobraking operations.

\section{Acknowledgments}

This work was sponsored by the NASA Engineering and Safety Center. Special thanks to Daniel Murri from the NESC for his guidance and support of this work. Team members who have significantly contributed to Phase 1 of the Autonomous Aerobraking assessment include Dr. Bobby Williams and Dave Skinner from Kinetx Aerospace for the Ephemeris Estimator model; Dr. Robert Tolson from the National Institute of Aerospace (and North Carolina State University) for the Atmosphere Estimator; John Dec and Mark Thornblom from NASA Langley Research Center for the spacecraft temperature modeling; Derek Liechty from NASA Langley Rearch Center and Forrest Lumpkin from NASA Johnson Space Center for aerodynamic and aerothermodynamic assessments; Jere Justus, Hilary Justh, and Holly Ramey from NASA Marshall Research Center for the "truth" atmosphere models used: MarsGRAM, VenusGRAM, and TitanGRAM; Chris Pastore from Analytical Mechanics Associates, Inc. for software development support; and Dan O'Shaughnessy, Jim Kaidy, David Carrelli, Hollis Ambrose, and Tom Strikwerda from the Applied Physics Laboratory at John Hopkins University who supported AADS development and testing by integrating AADS within the MESSENGER-based high-fidelity simulation, and who are also responsible for the development of the State Propagator to be used in Phase 2 development.

\section{References}

${ }^{1}$ Spencer, D. A., Tolson, R. H., “Aerobraking Cost and Risk”, Journal of Spacecraft and Rockets, Vol. 44, No. 6, Nov-Dec 2007, pp 1285-1293.

${ }^{2}$ Hanna, J. L., Tolson, R. H., “Approaches to Autonomous Aerobraking at Mars", AAS 01-387, AAS/AIAA Astrodynamics Specialist Conference, Quebec City, Canada. July 30-August 2, 2001.

${ }^{3}$ Lyons, Daniel T., "Aerobraking Automation Options." AAS-01-385, AAS/AIAA Astrodynamics Specialist Conference, Quebec City, CA, 2001. 
${ }^{4}$ Hanna, J.L., Tolson, R.H., Cianciolo, A.M.D, and Dec, J.A., “Autonomous Aerobraking at Mars”, 5th International ESA Conference on Guidance Navigation and Control Systems and Actuator and Sensor Product Exhibition, Frascati, Italy, October 22-25, 2002, ESA SP-516, February 2003.

${ }^{5}$ Willcockson, W. H. and Johnson, M. A., "Mars Odyssey Aerobraking: The First Step Towards Autonomous Aerobraking Operations", 2003 IEEE Aerospace Conference, Big Sky, MT. March 9-14, 2003.

${ }^{6}$ Jah, M. K., et al., "Mars Aerobraking Spacecraft State Estimation by Processing Inertial Measurement Unit Data", Journal of Guidnace, Control, and Dynamics, Vol. 31, No. 6, Nov-Dec 2008, pp 1802-1813.

${ }^{7}$ Brauer, G. L., et al., "Program To Optimize Simulated Trajectories (POST): Volume 1, Formulation Manual." Martin Marietta Corporation, 1990.

${ }^{8}$ Striepe, S.A., et al., "Program To Optimize Simulated Trajectories (POST II): Volume 2, Utilization Manual." Martin Marietta Corporation, 2004.

${ }^{9}$ Maddock, R. W., et al., "Implementation and Simulation Results Using Autonomous Aerobraking Development Software", AAS 11-476, AAS/AIAA Astrodynamics Specialist Conference, Girdwood, AK, 2011.

${ }^{10}$ Prince, J. L. H., et al., "Development of Autonomous Aerobraking (Phase 1)", NASA Engineering and Safety Center Technical Assessment Report, NESC-RP-09-00605, December 15, 2011.

${ }^{11}$ Prince, J. L. H., Striepe, S. A., "NASA Langley Simulation Capabilities for the Mars Reconnaissance Orbiter", AAS/AIAA Space Flight Mechanics Conference, Copper Mountain, Colorado, January 23-27, 2005.

${ }^{12}$ Prince, J. L. H., Tolson, R. H., “Onboard Atmospheric Modeling and Prediction for Autonomous Aerobraking Missions", AAS 11-477, AAS/AIAA Astrodynamics Specialist Conference, Girdwood, AK, 2011.

${ }^{13}$ Dec, J. A., Thornblom, M. N., "Autonomous Aerobraking: Thermal Analysis and Response Surface Development", AAS 11-474, AAS/AIAA Astrodynamics Specialist Conference, Girdwood, AK, 2011. 